\title{
Hard X-Ray-selected AGNs in Low-mass Galaxies from the NuSTAR Serendipitous Survey
}

\author{
C.-T. J. Chen (陳建廷) ${ }^{1,2}$, W. N. Brandt ${ }^{1,2,3}$, A. E. Reines ${ }^{4,20}$, G. Lansbury ${ }^{5}$, D. Stern ${ }^{6}$, D. M. Alexander ${ }^{7}$, F. Bauer ${ }^{8,9,10}$, \\ A. Del Moro ${ }^{11}$, P. Gandhi ${ }^{12}$, F. A. Harrison ${ }^{13}$, R. C. Hickox ${ }^{14}$, M. J. Koss ${ }^{15,21}$, L. Lanz ${ }^{14}$, B. Luo ${ }^{16}$, J. R. Mullaney ${ }^{17}$, \\ C. Ricci $^{8,9,18}$, and J. R. Trump ${ }^{19}$ \\ ${ }^{1}$ Department of Astronomy and Astrophysics, Pennsylvania State University, University Park, PA 16802, USA; ctchen@psu.edu \\ ${ }^{2}$ Institute for Gravitation and the Cosmos, Pennsylvania State University, University Park, PA 16802, USA \\ ${ }_{4}^{3}$ Department of Physics, Pennsylvania State University, University Park, PA 16802, USA \\ ${ }^{4}$ National Optical Astronomy Observatory, 950 N. Cherry Ave., Tucson, AZ 85719, USA \\ ${ }^{5}$ Institute of Astronomy, University of Cambridge, Madingley Road, Cambridge, CB3 OHA, UK \\ ${ }^{6}$ Jet Propulsion Laboratory, California Institute of Technology, Pasadena, CA 91109, USA \\ ${ }^{7}$ Centre for Extragalactic Astronomy, Department of Physics, Durham University, South Road, Durham, DH1 3LE, UK \\ ${ }^{8}$ Instituto de Astrofísica and Centro de Astroingeniería, Facultad de Física, Pontificia Universidad Católica de Chile, Casilla 306, Santiago 22, Chile \\ ${ }^{9}$ Millennium Institute of Astrophysics (MAS), Chile \\ ${ }^{10}$ Space Science Institute, 4750 Walnut Street, Suite 205, Boulder, CO 80301, USA \\ ${ }^{11}$ Max-Planck-Institut für Extraterrestrische Physik (MPE), Postfach 1312, D-85741, Garching, Germany \\ 12 Department of Physics and Astronomy, University of Southampton, Highfield, Southampton SO17 1BJ, UK \\ ${ }_{13}^{13}$ Cahill Center for Astronomy and Astrophysics, California Institute of Technology, Pasadena, CA 91125, USA \\ ${ }^{14}$ Department of Physics and Astronomy, Dartmouth College, 6127 Wilder Laboratory, Hanover, NH 03755, USA \\ ${ }^{15}$ Institute for Astronomy, Department of Physics, ETH Zurich, Wolfgang-Pauli-Strasse 27, CH-8093 Zurich, Switzerland \\ ${ }^{16}$ School of Astronomy and Space Science, Nanjing University, Nanjing 210093, China \\ ${ }^{17}$ Department of Physics and Astronomy, The University of Sheffield, Hounsfield Road, Sheffield S3 7RH, UK \\ ${ }^{18}$ Kavli Institute for Astronomy and Astrophysics, Peking University, Beijing 100871, China \\ ${ }^{19}$ Department of Physics, University of Connecticut, 2152 Hillside Road, Storrs, CT 06269, USA \\ Received 2016 October 11; revised 2017 January 24; accepted 2017 January 27; published 2017 March 1
}

\begin{abstract}
We present a sample of 10 low-mass active galactic nuclei (AGNs) selected from the 40-month Nuclear Spectroscopic Telescope Array (NuSTAR) serendipitous survey. The sample is selected to have robust NuSTAR detections at 3-24 keV, to be at $z<0.3$, and to have optical $r$-band magnitudes at least 0.5 mag fainter than an $L_{\star}$ galaxy at its redshift. The median values of absolute magnitude, stellar mass, and 2-10 X-ray luminosity of our sample are $\left\langle M_{r}\right\rangle=-20.03,\left\langle M_{\star}\right\rangle=4.6 \times 10^{9} M_{\odot}$, and $\left\langle L_{2-10 \mathrm{keV}}\right\rangle=3.1 \times 10^{42} \mathrm{erg} \mathrm{s}^{-1}$, respectively. Five objects have detectable broad $\mathrm{H} \alpha$ emission in their optical spectra, indicating black hole masses of $(1.1-10.4) \times 10^{6} M_{\odot}$. We find that $30_{-10}^{+17} \%$ of the galaxies in our sample do not show AGN-like optical narrow emission lines, and one of the 10 galaxies in our sample, J115851+4243.2, shows evidence for heavy X-ray absorption. This result implies that a non-negligible fraction of low-mass galaxies might harbor accreting massive black holes that are missed by optical spectroscopic surveys and $<10 \mathrm{keV} \mathrm{X-ray} \mathrm{surveys.} \mathrm{The} \mathrm{mid-IR} \mathrm{colors} \mathrm{of}$ our sample also indicate that these optically normal low-mass AGNs cannot be efficiently identified with typical AGN selection criteria based on Wide Field Infrared Survey Explorer colors. While the hard $(>10 \mathrm{keV}) \mathrm{X}$-rayselected low-mass AGN sample size is still limited, our results show that sensitive NuSTAR observations are capable of probing faint hard X-ray emission originating from the nuclei of low-mass galaxies out to moderate redshift $(z<0.3)$, thus providing a critical step in understanding AGN demographics in low-mass galaxies.
\end{abstract}

Key words: galaxies: active - galaxies: dwarf - X-rays: galaxies

Supporting material: figure set

\section{Introduction}

Understanding the properties of the massive black holes (mBHs) in the centers of low-mass galaxies $\left(M_{\star} / M_{\odot} \lesssim 10^{10}\right)$ is an important way to discriminate observationally between different BH-seed formation scenarios (e.g., Volonteri 2010; Greene 2012; Reines \& Comastri 2016). It is also unclear whether the well-known scaling relation between the supermassive black hole (SMBH) mass and the velocity dispersion of the host-galaxy bulge extends to the low-mass regime (see Kormendy \& Ho 2013, for a review; but also see Barth et al. 2005; Xiao et al. 2011; Baldassare et al. 2015, 2016). Therefore, our understanding of galaxy evolution remains

\footnotetext{
${ }^{20}$ Hubble Fellow.

21 Ambizione Fellow.
}

incomplete without a clear picture of the $\mathrm{mBH}$ population in low-mass galaxies.

Active galactic nucleus (AGN) emission powered by accretion onto $\mathrm{mBHs}$ in low-mass galaxies is often diluted and/or mimicked by stellar processes in the host galaxies (e.g., Moran et al. 2002, 2014; Trump et al. 2015). Therefore, the identification of accreting $\mathrm{mBHs}$ in low-mass galaxies is challenging. In practice, single-epoch spectroscopic observations at optical wavelengths have been the most efficient method for reliably finding unobscured AGNs while also obtaining the estimated virial mass of the $\mathrm{mBHs}$ (Greene \& Ho 2004, 2007b; Reines et al. 2013; Baldassare et al. 2015). However, this approach requires that AGN signatures are clearly visible (i.e., not obscured or diluted) in the optical spectrum. Limited by the luminosity of such $\mathrm{mBH}$ accretion 
and current optical spectroscopic survey limits, most of the optically selected $\mathrm{mBH}$ candidates are unobscured AGNs at low redshifts (e.g., $z \lesssim 0.35$; Greene \& Ho 2007b; Reines et al. 2013).

On the other hand, X-ray stacking analyses of high-redshift galaxies in survey regions with deep X-ray observations have suggested that many low-mass galaxies harbor X-ray-emitting nuclei that are heavily obscured (Xue et al. 2012; Mezcua et al. 2016). To date, there are very few known AGNs residing in low-mass star-forming galaxies in the local universe (see Reines et al. 2011, 2014, for individual examples). This might be due to the fact that X-ray observations probing energies $<10 \mathrm{keV}$ can suffer from obscuration, as well as could be affected by galaxy dilution due to the low-luminosity nature of $\mathrm{mBH}$ accretion (Reines et al. 2016). Therefore, our understanding of X-ray-selected AGN demographics in low-mass galaxies is primarily limited to a few sources from survey regions with deep soft ${ }^{22} \mathrm{X}$-ray observations (E-CDF-S, Schramm et al. 2013; XDEEP2, Pardo et al. 2016; AMUSE, Gallo et al. 2008; Miller et al. 2015) or archival searches (Lemons et al. 2015). While there are also a number of soft $\mathrm{X}$-ray follow-up observations of mBHs selected using broad emission lines (e.g., Greene \& Ho 2007a; Dong et al. 2012a; Baldassare et al. 2017), most of them are X-ray unobscured as a consequence of the optical broad-line selection. Furthermore, Plotkin et al. (2016) and Simmonds et al. (2016) have reported that some AGNs with broad optical emission lines in low-mass galaxies have surprisingly weak $<10 \mathrm{keV}$ X-ray emission compared to the expected values from their [O III] and UV luminosities (Baldassare et al. 2017).

An alternative approach to studying the AGN populations hosted by low-mass galaxies is to observe in hard X-rays since hard X-ray photons are much less susceptible to absorption by intervening material. In addition, recent Nuclear Spectroscopic Telescope Array (NuSTAR; Harrison et al. 2013) studies of two star-forming galaxies have also revealed that the broadband X-ray spectra of galaxies powered by stellar processes are dominated by $k T \approx 0.2-1 \mathrm{keV}$ plasma emission at $E<1-3 \mathrm{keV}$ and a steep $(\Gamma \gtrsim 2.6-2.7)$ power-law component at $E>5-7 \mathrm{keV}$ (e.g., Lehmer et al. 2015). Notably, the power-law component of some of the most luminous ULXs (ultraluminous X-ray sources) could have photon indices similar to those of AGNs (e.g., $\Gamma \geqslant 1.4$; Walton et al. 2014; Mukherjee et al. 2015). Therefore, the combination of hard X-ray observations from $N u S T A R$ and high angular resolution data from ancillary soft X-ray observations can provide the means to distinguish the $\mathrm{X}$-ray emission originating from $\mathrm{mBH}$ versus other off-nuclear stellar processes. Constructing a NuSTAR-selected AGN sample hosted by low-mass galaxies could provide a critical step to understanding the low-mass AGN population.

Low-mass AGNs detected by previous hard X-ray observatories are scarce, due to the limited sensitivity and angular resolution of the previous generation of instruments. Even with the all-sky coverage of the Swift/BAT survey (e.g., Koss et al. 2011), the number of low-mass AGNs is small, and the lowmass AGNs detected by Swift/BAT primarily are composed of nearby $(z<0.005)$ or luminous $\left(L_{14-195 \mathrm{keV}}>10^{43} \mathrm{erg} \mathrm{s}^{-1}\right)$ sources that might not be representative of low-mass AGNs generally. The recently launched $N U S T A R$ observatory provides

\footnotetext{
22 Throughout the paper, we refer to X-rays in the $<10 \mathrm{keV}$ energy range as "soft" and $\geqslant 10 \mathrm{keV}$ X-rays as "hard."
}

a $>100$ times improvement in hard X-ray sensitivity over previous observations at $\gtrsim 10 \mathrm{keV}$. One of the first 10 NuSTAR detections in the NuSTAR serendipitous survey (Alexander et al. 2013) has already been identified as an AGN hosted by a dwarf galaxy $\left(M_{\star} \approx 1.5 \times 10^{9} M_{\odot}\right)$. In this work, we report the properties of low-mass galaxies detected in the NuSTAR serendipitous-survey catalog (Lansbury et al. 2017, hereafter L17), aiming to improve our understanding of the hard X-ray-emitting AGN population hosted by low-mass galaxies.

This paper is organized as follows: In Section 2, we describe the selection of low-mass galaxies from the NuSTAR serendipitous survey. The data analysis is presented in Section 3. In Section 4, we study the AGN properties of our sample. In Section 5, we compare the multiwavelength properties of our NuSTAR sample with those of previous AGN samples hosted by low-mass galaxies. A discussion and summary are provided in Section 6. Detailed data analysis for individual objects is presented in Appendix A. Throughout the paper, we assume $H_{0}=70 \mathrm{~km} \mathrm{~s}^{-1} \mathrm{Mpc}^{-1}$ and a $\Lambda \mathrm{CDM}$ cosmology with $\Omega_{m}=0.3$ and $\Omega_{\Lambda}=0.7$. The uncertainties reported in this work are $1 \sigma$ unless stated otherwise.

\section{Sample Selection and Stellar-mass Estimation}

\subsection{The NuSTAR Serendipitous Survey}

To date, the NuSTAR serendipitous survey (L17) has covered an area of $\approx 13 \mathrm{deg}^{2}$ and detected 497 individual sources. The serendipitous survey is the largest-area component of the $N u S T A R$ extragalactic survey program. This program searches for serendipitous detections in most of the NUSTAR pointings. In short, the serendipitous catalog includes soft-band (NuSTAR$\mathrm{SB}, 3-8 \mathrm{keV})$ and hard-band (NuSTAR-HB, 8-24 keV) photometry for sources detected in the full band (NuSTAR-FB, 3-24 keV). Sources were extracted using the source-detection software package SExtractor (Bertin \& Arnouts 1996) on the false-positive probability $\left(P_{\text {False }}\right.$ hereafter) map generated from the mosaics of the two NuSTAR focal-plane modules (FPMA and FPMB). A more detailed discussion of source detection and the $P_{\text {False }}$ map generation can be found in Mullaney et al. (2015) and L17.

In this work, we focus on the 248 objects that are spectroscopically confirmed to be extragalactic. Their redshifts were primarily obtained from the dedicated spectroscopic follow-up observations as part of the NUSTAR serendipitous survey (see L17 for details), and also from publicly available spectroscopic surveys. We refer readers to L17 for details and a complete discussion of NuSTAR source-extraction methods and the NUSTAR serendipitous catalog.

\subsection{Multiwavelength Counterpart Identification}

Source matching and counterpart identification for NuSTAR are challenging, due to the relatively large point-spread function (PSF; $\approx 18^{\prime \prime}$ FWHM) and astrometric uncertainty $(\approx 6$." 6; see Civano et al. 2015). For faint sources, the positional uncertainty of NuSTAR can be as high as $\approx 22^{\prime \prime}$ (see Section 3.1 of L17). We first match each NUSTAR source position to the closest Chandra, XMM-Newton, or Swift/XRT source when available, as the positional accuracy of these lower-energy $\mathrm{X}$-ray observatories is better. We then match the soft X-ray positions to the Sloan Digital Sky Survey (SDSS; York et al. 2000) or the SuperCOSMOS scans of photographic 
Schmidt plates (Hambly et al. 2001) with a $5^{\prime \prime}$ search radius. We note that 15 of the 248 sources do not have ancillary soft X-ray observations. For these objects, we use a searching radius of $22^{\prime \prime}$ around the $N U S T A R$ positions to search for their optical counterparts. Based on visual inspection of the optical images, we reject seven of the 15 objects with multiple optical and infrared counterparts within the search radius to avoid potentially spurious matching results. Each of the other eight sources is matched to the only optical counterpart within the $22^{\prime \prime}$ search radius. We note that none of the 15 objects are included in our final sample of low-mass AGNs.

Of the 248 extragalactic $N u S T A R$ serendipitous sources, all have $r$-band photometry. A total of 113 of the 248 objects are covered in the imaging footprint of SDSS, and all of these objects have optical photometry in the $u, g, r, i, z$ bands. To measure accurately the host-galaxy color and luminosity, we adopt the extinction-corrected model magnitudes that are scaled to the $i$-band c-model magnitude ${ }^{23}$ from SDSS DR12 (Alam et al. 2015).

For the 135 objects outside of the SDSS imaging footprint, the optical photometry was obtained from SuperCOSMOS, which is considered to have 0.3 mag photometric uncertainties.

Near-IR constraints for our sample come from the $J$-, $H$-, and $K_{s}$-band photometric catalog of the Two Micron All Sky Survey (2MASS; Skrutskie et al. 2006). We also obtain mid-IR photometry from the ALLWISE catalog, which is an all-sky catalog covering the $3.4,4.6,12$, and $22 \mu \mathrm{m}$ bands (W1, W2, W3, and W4 hereafter) observed with the Wide Field Infrared Survey Explorer (WISE; Wright et al. 2010). We make use of the UV GALEX (Martin et al. 2005) photometry from the GALEX Release 6/7 (Bianchi et al. 2014). We correct for the Galactic extinction in the GALEX near- and far-UV bands using the $E(B-V)$ values from the GALEX catalog and the $R_{V}$ values from Wyder et al. (2007). For the optical, near-IR, and mid-IR bands, the Galactic extinction values were obtained using the IRSA Galactic Reddening and Extinction Calculator using the Schlegel et al. (1998) extinction map. Counterparts in the UV, near-IR, and mid-IR bands were obtained by searching around the optical positions within a $5^{\prime \prime}$ radius. We note that three of the low-redshift serendipitous objects appear to be extended in the near-IR and mid-IR images (J023229+2023.7, $\mathrm{J} 032459-0256.2$, and $\mathrm{J} 115851+4243.2$; see Figure set 2 below) and the default profile-fit photometry provided by 2MASS and ALLWISE might not be optimal. To obtain accurate photometry for these three extended sources, we adopt the $20 \mathrm{mag} \mathrm{deg}^{-2}$ isophotal fiducial elliptical magnitudes in the 2MASS $J, H$, and $K_{s}$ bands and the WISE magnitudes measured via elliptical aperture photometry. All 248 objects have a signal-to-noise ratio $(\mathrm{S} / \mathrm{N})>5$ in the WISE W1 and W2 bands. With the more limited sensitivity of 2MASS and GALEX, only 80 objects are detected in the $J, H$, and $K_{s}$ bands, and only 114 objects are detected by $G A L E X$.

\subsection{The NuSTAR Low-mass Galaxy Sample}

To construct a sample of low-mass galaxies with robust detections in hard X-rays, we focus on a low-redshift $(z<0.3)$ subsample of the NUSTAR serendipitous-survey sources covered by both FPMA and FPMB and having $|b|>10^{\circ}$. The redshift cut is motivated by the sensitivity limit of NuSTAR. Beyond $z \approx 0.3$, even for an $\mathrm{mBH}$ with $M_{\bullet}=10^{7} M_{\odot}$

\footnotetext{
${ }^{23}$ http://www.sdss.org/dr12/spectro/galaxy_portsmouth/
}

radiating at the Eddington limit $\left(L_{10-40 \mathrm{keV}} \approx 6 \times 10^{43} \mathrm{erg}\right.$ $\left.\mathrm{s}^{-1}\right)$, the expected count rates in the NuSTAR-HB $(8-24 \mathrm{keV})$ would still be too low for $N u S T A R$ to provide detections in the typical exposure times of the NuSTAR serendipitous survey (the serendipitous survey has a median exposure time of $28 \mathrm{ks}$; see L17).

We next search for candidate low-mass galaxies by comparing the extinction-corrected $r$-band magnitude (observed-frame AB magnitudes) with that of an $L_{\star}$ galaxy (i.e., a galaxy with a luminosity equal to the value of the "knee" of the luminosity function) calculated using the Kelvin et al. (2014) luminosity function for elliptical galaxies. There are a total of 10 sources at $z<0.3$ with an $r$-band magnitude that is at least 0.5 mag fainter than $r_{\star}$ ( $r$-band magnitude of an $L_{\star}$ galaxy) at the corresponding redshift.

L17 report a spurious matching rate of $\approx 7 \%$ between the NUSTAR and soft X-ray positions. To ensure that the NUSTAR positions of the low-mass galaxy candidates are matched to the correct counterpart, we visually inspected the soft X-ray, optical, near-IR, and mid-IR images for each of the 10 objects. We find that nine of the 10 sources have only one soft X-ray counterpart within a $22^{\prime \prime}$ radius. The other object, J115851 +4243.2 , has three soft X-ray point sources within a $22^{\prime \prime}$ radius, but only the source at the galactic center has a $3-8 \mathrm{keV}$ flux comparable to the NuSTAR 3-8 keV flux. We adopt the central source as the NuSTAR counterpart. Therefore, we consider the matching between the NUSTAR positions and the soft X-ray positions to be accurate. For each source, the soft $\mathrm{X}$-ray position has only one optical counterpart within a $5^{\prime \prime}$ search radius.

As a result, we have a final sample of 10 low-mass galaxy candidates with NUSTAR detections. This sample-selection approach is chosen because the stellar-mass measurements are often sensitive to the choice of initial mass function and the uncertainty of stellar population synthesis models used to estimate $M_{\star}$. For AGN host galaxies, the stellar-mass estimation is further complicated by the AGN emission. We note that, by happenstance, our empirical selection criterion of $r<r_{\star}-0.5$ recovers all of the $M_{\star}<10^{10} M_{\odot}$ galaxies detected in the NUSTAR serendipitous catalog at $z<0.3$ (see Section 3.1 for the details of stellar-mass estimation for the $N U S T A R$ serendipitous catalog). We show the redshift versus $r$ band magnitude distribution for our low-mass galaxy sample in Figure 1(a).

All 10 galaxies in our sample satisfy the $\log P_{\mathrm{FALSE}}<-6$ significance criterion in the $3-24 \mathrm{keV}$ band (L17). In NuSTAR$\mathrm{HB}$, only two objects in our sample pass the same falsedetection probability criterion. Since the objects in our sample were carefully matched to their soft X-ray to mid-IR counterparts, it is less likely for the NuSTAR detections at these positions to be caused by random Poisson noise. Thus, we consider the eight objects with $>2 \sigma$ net counts in the $8-24 \mathrm{keV}$ band to be reliably detected in NuSTAR-HB. For the other two objects with $N u S T A R$-HB net counts less than $2 \sigma$, we consider these objects as nondetected. We adopt the gross source count (background plus source counts) uncertainty estimated using the Gehrels (1986) method as the HB net count upper limits for these objects without HB detections. The NuSTAR low-mass AGNs are presented in Table 1. Their NuSTAR photometric properties are listed in Table 2, and their multiwavelength properties are listed in Table 3. Since the NuSTAR positional 


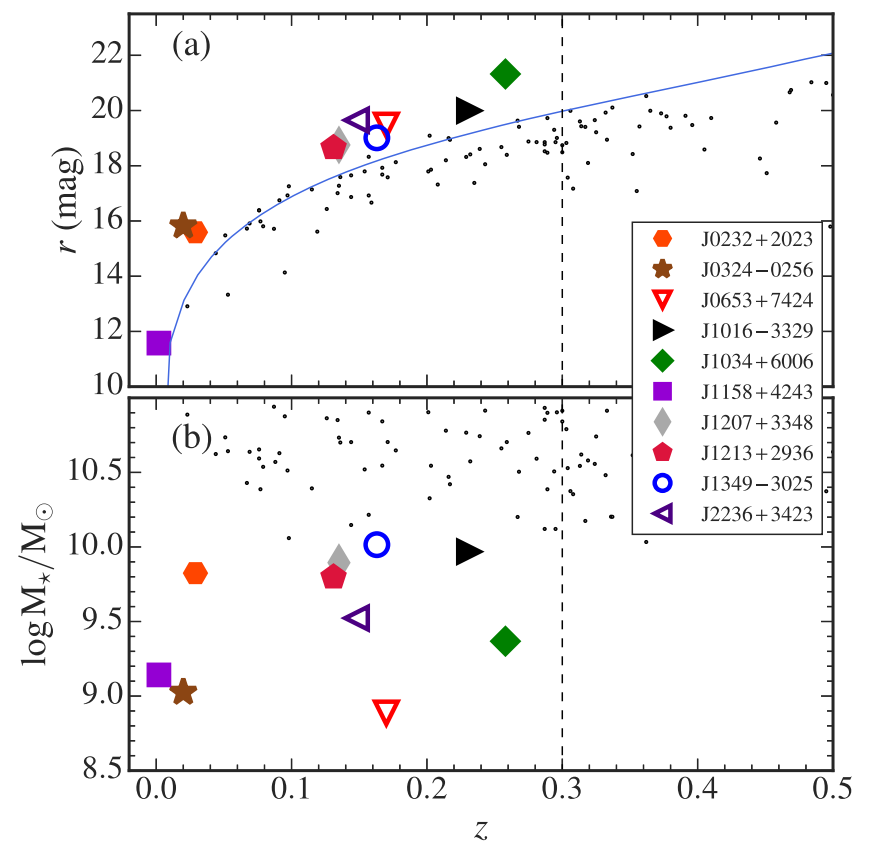

Figure 1. Redshift as a function of (a) $r$-band AB magnitudes and (b) $M_{\star}$ for the $z<0.3 N u S T A R$ low-mass AGN sample. The dashed line marks the $z=0.3$ redshift cut. Our sample is selected to be at least 0.5 mag fainter than an $L_{\star}$ galaxy at the corresponding redshift. The observed-frame $r$-band luminosity of an $L_{\star}$ galaxy derived from the Kelvin et al. (2014) luminosity function is plotted as the solid line in panel (a). The full spectroscopic sample at $z<0.5$ from the NuSTAR serendipitous survey is shown as gray dots.

uncertainty is $\sim 22^{\prime \prime}$, we also list the angular offset between the NuSTAR positions and the optical positions in Table 3.

\section{Data Analysis}

In this section, we outline data-analysis methods and results for the NUSTAR low-mass galaxy sample. For each object, we use a spectral energy distribution (SED) fitting analysis to estimate its stellar mass in Section 3.1. The NUSTAR and ancillary soft X-ray data are presented in Sections 3.2 and 3.3, respectively. The optical spectral analysis results are presented in Section 3.4. The data-analysis results for individual objects are shown in the online figure set (Figure 2) and Appendix A.

\subsection{SED Fitting and Stellar-mass Estimation}

To estimate $M_{\star}$ for the members of the low-mass AGN sample, we use an SED-fitting based approach similar to the one adopted by Alexander et al. (2013), which takes the possible contribution from the AGN into account. We fit the photometry spanning the UV to mid-IR with the low-resolution templates from Assef et al. (2010), which are composed of three galaxy templates and one AGN template. This approach has been shown to be able to reproduce accurately the SEDs of a wide variety of galaxies (see Assef et al. 2008, for details), as well as effectively disentangle the AGN contribution from the host-galaxy photometry (e.g., Chung et al. 2014; Stern et al. 2014; Chen et al. 2015; Lansbury et al. 2015). For the AGN component, we create a grid of AGN templates with $0<E(B-V)<10$ by applying dust reddening to the Assef et al. (2010) AGN template. The extinction model we use is a hybrid of an SMC-like (Small Magellanic Cloud) extinction curve at $\lambda<3300 \AA$ (Gordon \& Clayton 1998) and a Galactic extinction curve at longer wavelengths (Cardelli et al. 1989), with $R_{V}=3.1$ for both. This is motivated by the observed lack of the $2175 \AA$ carbon feature in QSO spectra (see York et al. 2006; Assef et al. 2010, for details). Once the best-fit SED is determined using the non-negative least-squares algorithm presented by Assef et al. (2010), we calculate the rest-frame $i$ band absolute magnitude and rest-frame $r-z$ color of the galaxy component of the best-fitting SED, which is then used to estimate the stellar mass using the color-dependent mass-tolight ratio described in Zibetti et al. (2009), with a Chabrier initial mass function. We present the resulting $M_{\star}$ values of the NuSTAR AGN hosted by low-mass galaxies as a function of redshift in Figure 1(b). For comparison, we have also shown the $M_{\star}$ values for the parent $N u S T A R$ serendipitous sample with spectroscopic redshift $z<0.5$ in the same figure.

For the seven NuSTAR low-mass AGN candidates with SDSS photometry, we also calculate $M_{\star}$ using the KCORRECT package $^{24}$ (Blanton et al. 2003) without correcting for the AGN contribution. For our sample, the SED-fitting estimated $M_{\star}$ is only slightly lower than the KCORRECT $M_{\star}$ by $\approx 0.04$ dex, which is consistent with previous studies that suggest that the AGN contamination at optical to near-IR wavelengths is generally not significant for local low-luminosity broad-line AGNs (Reines \& Volonteri 2015; Hainline et al. 2016). In this work, we adopt the $M_{\star}$ values estimated using the SED-fitting method described above for the low-mass AGN candidates in our sample. The errors on $M_{\star}$ are expected to be dominated by $\approx 0.3$ dex uncertainties associated with the stellar population synthesis model degeneracy (Conroy et al. 2009). We list the stellar masses in Table 3, and the SED-fitting results for each object are shown in Figure 2. The stellar-mass range for the 10 galaxies in our final sample is $8.9-10.0\left(\log M_{\odot}\right)$.

\subsection{Basic NuSTAR Properties}

Due to the design of the NUSTAR serendipitous survey, the low-mass galaxies in our sample are covered by NuSTAR observations with a wide range of exposure times, between 12 and $125 \mathrm{ks}$ (see Table 2 for the NUSTAR properties of our sample). Some of them have substantial background counts (see Table 2), due to small angular separation from the luminous primary science targets. Some of the sources are located at the edge of the NUSTAR field of view (FOV), thus making spectral extraction challenging. Only four of the sources in our sample have sufficient net photon counts and manageable background to be suitable for X-ray spectralfitting analyses (J023229+2023.7, J032459-0256.2, J065318 +7424.8, and J101609-3329.6). In the L17 serendipitous catalog, an observed hard X-ray luminosity has been calculated for each object in the rest-frame $10-40 \mathrm{keV}$ band $\left(L_{10-40 \mathrm{keV}}\right.$ hereafter) using the NuSTAR hard-band $(8-24 \mathrm{keV})$ flux derived from the count rates with a count rate to flux conversion factor based on a power-law AGN spectrum with a photon index of $\Gamma=1.8$. There are two objects with less than $2 \sigma$ detection significance in the hard band. We consider their $L_{10-40 \mathrm{keV}}$ to be upper limits. For the other objects with NuSTAR-HB detections, their NuSTAR-HB flux uncertainties translate into an $L_{10-40 \mathrm{keV}}$ average uncertainty of 0.2 dex. Since the photon counts for our low-mass AGN candidates are limited, we use the band ratio $(H / S$, in which $S$ and $H$ represent the NuSTAR net counts in the 3-8 keV and 8-24 keV bands) to estimate the basic X-ray spectral shape for each source,

\footnotetext{
${ }^{24}$ http://kcorrect.org/
} 
Table 1

NUSTAR Low-mass Galaxies

\begin{tabular}{lllrr}
\hline \hline $\begin{array}{l}\text { ID } \\
(1)\end{array}$ & $\begin{array}{c}\text { Source Name } \\
(2)\end{array}$ & $\begin{array}{c}\text { Target Field } \\
(3)\end{array}$ & $\begin{array}{c}\text { R.A. (J2000) } \\
(4)\end{array}$ & $\begin{array}{r}\text { Decl. (J2000) } \\
(5)\end{array}$ \\
\hline 1 & J023229+2023.7 & 1ES 0229+200 & $\begin{array}{c}z \\
(6)\end{array}$ \\
2 & J032459-0256.2 & NGC 1320 & 38.120106 & 20.396729 \\
3 & J065318+7424.8 & Mrk 6 & 51.249614 & -2.9364875 \\
4 & J101609-3329.6 & IC2560 & 103.331370 & 74.418452 \\
5 & J103410+6006.7 & Mrk 34 & 154.003625 & -33.493796 \\
6 & J115851+4243.2 & IC 751 & 158.541966 & 60.112053 \\
7 & J120711+3348.5 & WAS 49b & 179.713759 & 42.721351 \\
8 & J121358+2936.1 & IC 4329A & 181.797028 & 33.807852 \\
9 & J134934-3025.5 & SN 2014C & 183.494820 & 0.170 \\
10 & J223654+3423.5 & 207.39206 & 0.231 \\
& & & 339.226251 & 0.002 \\
\end{tabular}

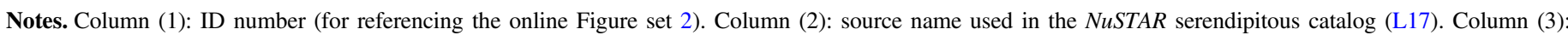

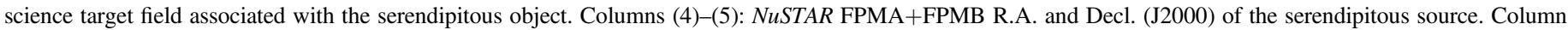
(6): redshift.

a Also known as SDSS J1034+6001 in Gandhi et al. (2014).

b Also known as IC 750.

respectively. To account for the uncertainties associated with the high background counts of NuSTAR observations, we calculate the band ratios using the Bayesian Estimation of Hardness Ratios method (BEHR; Park et al. 2006). The calculated band ratios for our low-mass AGN candidates span a range of 0.43-1.3 (see Table 2). We used the XSPEC FAKEIT command to simulate NUSTAR spectra and found that this range of band ratios corresponds to spectra with effective power-law photon indices in the range $0.9<\Gamma<2.5$. With the substantial uncertainties due to the limited photon statistics, limited energy range, and high background, the NuSTAR band ratios alone do not allow us to determine whether there is substantial obscuration in any of the low-mass AGNs. Thus, we utilize the ancillary soft X-ray observations to explore further the X-ray spectral properties of our low-mass AGNs in the following subsections. The NuSTAR band ratios and $L_{10-40 \mathrm{keV}}$ values are listed in Table 2 . For our sample, the $L_{10-40 \mathrm{keV}}$ range is 39.8-43.4 (in logarithmic erg s${ }^{-1}$ ) and the 3-24 keV flux range is $(6.2-117.1) \times 10^{-14} \mathrm{erg} \mathrm{cm}^{-2} \mathrm{~s}^{-1}$.

\subsection{Ancillary X-Ray Observations and 2-10 keV $X$-Ray Luminosities}

We use existing data from other X-ray observatories to help constrain the X-ray spectral properties of the NUSTAR lowmass galaxies. For the 10 objects in our sample, seven of them have archival XMM-Newton data.

We use the Science Analysis Software (SAS v.15.0.0 ${ }^{25}$ ) to process the XMM-Newton Observation/Slew Data Files downloaded from the XMM-Newton science archive. ${ }^{26}$ Each observation is processed with the SAS task epicproc using the latest calibration files (as of December 2016). High-energy light curves are generated from the EPIC event files (10-12 keV for PN and $>10 \mathrm{keV}$ for MOS) and then used for screening background flares. Source spectra are extracted from the background-filtered event files using a circular region with a radius of $\approx 10^{\prime \prime}-20^{\prime \prime}$. Background spectra are extracted using circular source-free regions next to the corresponding source $\left(\approx 30^{\prime \prime}-60^{\prime \prime}\right.$ radius regions). Using the SAS tasks rmfgen and arfgen, we also produced the response

\footnotetext{
25 http://www.cosmos.esa.int/web/xmm-newton/sas-download

26 http://nxsa.esac.esa.int
}

matrices for each source for the EPIC pn detector. We note that several objects in our sample fall outside of the FOVs of the EPIC MOS detectors, and thus we only adopt the data from the pn detector for consistency.

There are also three objects with publicly available Chandra observations. Their data were analyzed using CIAO 4.8. The data were reprocessed using the chandra_repro pipeline to create the new level 2 event files. The Chandra source spectra were extracted from circular regions with a radius of $\approx 2^{\prime \prime}-10^{\prime \prime}$, while the background spectra were extracted from several source-free regions of $\approx 40^{\prime \prime}$ radius, selected at different positions around the source to account for local background variations.

For J032459-0256.2 and J120711+3348.5, we obtained the contemporaneous archival Swift/XRT data and used the HEAsoft (v.6.12) pipeline $^{27}$ xrtpipeline for data reduction. This cleans the event files using appropriate calibration files and extracts the spectra and ancillary files for a given source position; the source-extraction regions had radii of $\approx 20^{\prime \prime}$.

The data quality varies substantially between different objects, and only seven of the sources in our sample have enough photon counts for X-ray spectral fitting. For these seven galaxies $\quad(\mathrm{J} 023229+2023.7, \quad \mathrm{~J} 032459-0256.2, \quad \mathrm{~J} 065318$ $+7424.8, \quad \mathrm{~J} 101609-3329.6, \quad \mathrm{~J} 103410+6006.7, \quad \mathrm{~J} 115851$ +4243.2 , and $\mathrm{J} 121358+2936.1$ ), we use XSPEC $12.9 .0^{28}$ to perform spectral analysis of the unbinned data using the Cash statistic (Cash 1979). For the four galaxies with acceptable NUSTAR data, we jointly fit the NUSTAR and soft X-ray data in our spectral analysis. For each object, we start the analysis by fitting the data with a basic absorbed power-law model using TBABS ${ }^{*}$ (ZTBABS ${ }^{*}$ ZPOW) from XSPEC, which takes both intrinsic and Galactic absorption column densities into account. We then use the XSPEC goodness command to assess the goodness of fit. The goodness command was set to simulate 1000 spectra from the best-fit parameters. For each simulated spectrum, goodness computes the Kolmogorov-Smirnov (K-S) statistic, the "similarity" between the model spectra and the data, and the similarity between the best-fit model and the

\footnotetext{
27 http://heasarc.gsfc.nasa.gov/docs/software/lheasoft/

28 https://heasarc.gsfc.nasa.gov/xanadu/xspec/
} 


\begin{tabular}{|c|c|c|c|c|c|c|c|c|c|c|c|c|}
\hline $\begin{array}{l}\text { Source Name } \\
\text { (1) }\end{array}$ & $\begin{array}{l}\text { OBSID } \\
\text { (2) }\end{array}$ & $\begin{array}{c}\log P_{\text {False }} \\
\text { (3) }\end{array}$ & $\begin{array}{l}\text { Exp } \\
(\mathrm{ks}) \\
(4)\end{array}$ & $\begin{array}{c}\text { Flux } \\
(3-8 \mathrm{keV}) \\
(5)\end{array}$ & $\begin{array}{c}\text { Flux } \\
(8-24 \mathrm{keV}) \\
(6)\end{array}$ & $\begin{array}{c}\text { Flux } \\
(3-24 \mathrm{keV}) \\
(7)\end{array}$ & $\begin{array}{l}\text { Net Source } \\
\text { Counts } \\
(3-8 \mathrm{keV}) \\
(8)\end{array}$ & $\begin{array}{l}\text { Net Source } \\
\text { Counts } \\
(8-24 \mathrm{keV}) \\
(9)\end{array}$ & $\begin{array}{c}\text { Background } \\
\text { Counts } \\
(3-24 \mathrm{keV}) \\
(10)\end{array}$ & $\begin{array}{r}\text { Band Ratio } \\
\text { (11) }\end{array}$ & $\begin{array}{c}\log L_{10-40 \mathrm{keV}} \\
\left(\mathrm{erg} \mathrm{s}^{-1}\right) \\
(12)\end{array}$ & $\begin{array}{c}\log L_{10-40 \mathrm{keV}}^{\mathrm{Model}} \\
\left(\mathrm{erg} \mathrm{s}^{-1}\right) \\
(13)\end{array}$ \\
\hline $\mathrm{J} 023229+2023.7$ & 60002047 & -83.1 & 37.5 & $40.33 \pm 3.08$ & $71.62 \pm 7.29$ & $117.08 \pm 7.1$ & $275 \pm 21$ & $193 \pm 20$ & 279 & $0.68_{-0.07}^{+0.07}$ & 42.3 & $42.6(42.6)$ \\
\hline J032459-0256.2 & 60061036 & -49.8 & 12.0 & $36.07 \pm 5.08$ & $49.78 \pm 10.55$ & $94.6 \pm 10.81$ & $78 \pm 11$ & $43 \pm 9$ & 42 & $0.54_{-0.11}^{+0.1}$ & 41.8 & $41.7(41.7)$ \\
\hline J065318+7424.8 & 60102044 & -9.9 & 71.4 & $2.84 \pm 1.03$ & $6.14 \pm 2.46$ & $8.59 \pm 2.23$ & $34 \pm 12$ & $30 \pm 12$ & 187 & $0.86_{-0.33}^{+0.28}$ & 42.8 & $43.1(43.1)$ \\
\hline J101609-3329.6 & 50001039 & $-15.0^{\mathrm{a}}$ & 43.8 & $10.91 \pm 2.37$ & $12.07 \pm 5.4$ & $27.0 \pm 5.46$ & $76 \pm 16$ & $31 \pm 14$ & 146 & $0.35_{-0.11}^{+0.08}$ & 43.4 & $43.7(43.7)$ \\
\hline $\mathrm{J} 103410+6006.7$ & 60001134 & -9.1 & 25.0 & $4.67 \pm 1.94$ & $12.81 \pm 4.85$ & $16.26 \pm 4.38$ & $20 \pm 8$ & $23 \pm 9$ & 70 & $1.15_{-0.46}^{+0.35}$ & 43.4 & $42.9(42.9)$ \\
\hline $\mathrm{J} 115851+4243.2$ & 60001148 & -8.3 & 39.0 & $5.34 \pm 1.56$ & $7.88 \pm 3.99$ & $14.63 \pm 3.71$ & $38 \pm 11$ & $22 \pm 11$ & 145 & $0.45_{-0.21}^{+0.14}$ & 39.0 & $38.7(38.8)$ \\
\hline $\mathrm{J} 120711+3348.5$ & 60061356 & -6.1 & 14.8 & $10.07 \pm 3.63$ & $<12.29^{\mathrm{b}}$ & $25.53 \pm 7.72$ & $18 \pm 7$ & $<9^{\mathrm{b}}$ & 23 & $0.44_{-0.23}^{+0.16}$ & $<43.1^{\mathrm{b}}$ & $\cdots$ \\
\hline $\mathrm{J} 121358+2936.1$ & 60061335 & -14.9 & 16.1 & $10.46 \pm 2.91$ & $16.74 \pm 6.71$ & $29.24 \pm 6.48$ & $31 \pm 9$ & $20 \pm 10$ & 51 & $0.61_{-0.21}^{+0.18}$ & 43.0 & $42.2(42.2)$ \\
\hline J134934-3025.5 & 60001045 & -9.8 & 125.4 & $2.85 \pm 0.86$ & $6.91 \pm 2.11$ & $9.57 \pm 2.01$ & $65 \pm 19$ & $62 \pm 19$ & 534 & $0.99_{-0.35}^{+0.26}$ & 42.7 & $\cdots$ \\
\hline $\mathrm{J} 223654+3423.5$ & 80001085 & $-2.7^{\mathrm{a}}$ & 79.7 & $2.47 \pm 1.3$ & $<7.57^{\mathrm{b}}$ & $6.16 \pm 2.89$ & $27 \pm 14$ & $<35^{\mathrm{b}}$ & 189 & $1.3_{-0.53}^{+0.42}$ & $<42.8^{\mathrm{b}}$ & $\ldots$ \\
\hline
\end{tabular}

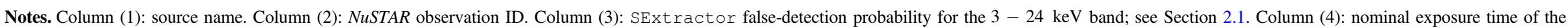

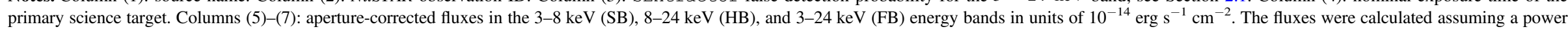

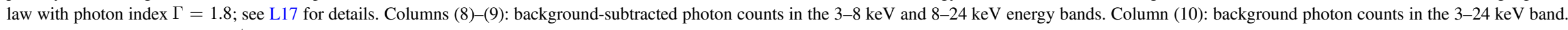

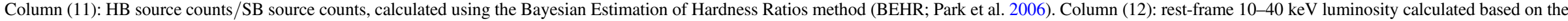

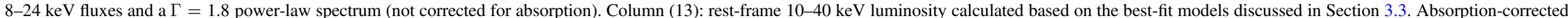

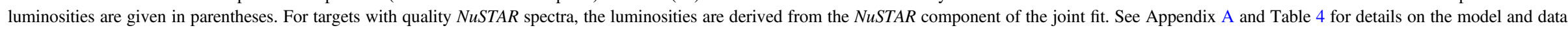
used for each source.

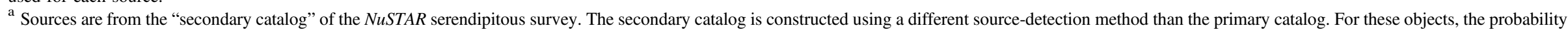
reported here is the binomial no-source probability; see L17 for details.

$\mathrm{b} 1 \sigma$ upper limits. 
Table 3

NUSTAR Source Photometry

\begin{tabular}{|c|c|c|c|c|c|c|c|c|c|c|}
\hline Name $^{a}$ & $\mathrm{~J} 023229+2023.7$ & J032459-0256.2 & J065318+7424.8 & J101609-3329.6 & $\mathrm{J} 103410+6006.7$ & $\mathrm{~J} 115851+4243.2$ & $\mathrm{~J} 120711+3348.5$ & $\mathrm{~J} 121358+2936.1$ & $\mathrm{~J} 134934-3025.5$ & $\mathrm{~J} 223654+3423.5$ \\
\hline R.A. $(\mathrm{J} 2000)^{\mathrm{b}}$ & 38.120017 & 51.249724 & 103.337760 & 154.00501 & 158.543011 & 179.71751 & 181.796853 & 183.497104 & 207.388010 & 339.224396 \\
\hline Decl. $(\mathrm{J} 2000)^{\mathrm{b}}$ & 20.397181 & -2.936738 & 74.417266 & -33.492958 & 60.112193 & 42.72247 & 33.811587 & 29.604993 & -30.426759 & 34.392549 \\
\hline Optical offset $^{\mathrm{c}}$ & 1.6 & 1.0 & 7.5 & 5.1 & 1.9 & 10.7 & 13.4 & 11.7 & 12.8 & 7.3 \\
\hline GALEX far-UV & $\ldots$ & $19.06 \pm 0.12$ & $\ldots$ & $\ldots$ & $22.90 \pm 0.13$ & $17.85 \pm 0.07$ & $20.75 \pm 0.21$ & $20.95 \pm 0.10$ & $23.28 \pm 0.32$ & $\ldots$ \\
\hline GALEX near-UV & $\cdots$ & $18.60 \pm 0.06$ & $\ldots$ & $\ldots$ & $\ldots$ & $\ldots$ & $20.74 \pm 0.13$ & $20.54 \pm 0.05$ & $22.50 \pm 0.12$ & $23.08 \pm 0.26$ \\
\hline$u^{\mathrm{d}}$ & $17.84 \pm 0.03$ & $\ldots$ & $\ldots$ & $\ldots$ & $23.49 \pm 0.69$ & $14.64 \pm 0.01$ & $20.08 \pm 0.04$ & $19.7 \pm 0.04$ & $\ldots$ & $21.47 \pm 0.37$ \\
\hline$g / B^{\mathrm{d}}$ & $16.38 \pm 0.01$ & 16.59 & 21.57 & $\cdots$ & $22.27 \pm 0.1$ & $12.57 \pm 0.01$ & $19.44 \pm 0.01$ & $19.16 \pm 0.01$ & 20.3 & $20.54 \pm 0.04$ \\
\hline$r / R^{\mathrm{d}}$ & $15.59 \pm 0.01$ & 15.95 & 19.77 & 20.2 & $21.32 \pm 0.08$ & $11.58 \pm 0.01$ & $18.75 \pm 0.01$ & $18.65 \pm 0.01$ & 19.13 & $19.65 \pm 0.03$ \\
\hline$i / I^{\mathrm{d}}$ & $15.21 \pm 0.01$ & 15.41 & $\cdots$ & 19.02 & $20.96 \pm 0.08$ & $11.06 \pm 0.01$ & $18.32 \pm 0.01$ & $18.18 \pm 0.01$ & 18.44 & $19.19 \pm 0.03$ \\
\hline$z^{\mathrm{d}}$ & $14.93 \pm 0.01$ & $\cdots$ & $\cdots$ & $\cdots$ & $20.94 \pm 0.34$ & $10.62 \pm 0.01$ & $18.08 \pm 0.02$ & $18.11 \pm 0.04$ & $\cdots$ & $18.59 \pm 0.08$ \\
\hline$J^{\mathrm{d}}$ & $13.89 \pm 0.05$ & $14.23 \pm 0.06$ & $\ldots$ & $\ldots$ & $\ldots$ & $9.28 \pm 0.01$ & $16.85 \pm 0.16$ & $17.02 \pm 0.22$ & $\ldots$ & $\ldots$ \\
\hline$H^{\mathrm{d}}$ & $13.18 \pm 0.06$ & $13.63 \pm 0.08$ & $\ldots$ & $\ldots$ & $\ldots$ & $8.5 \pm 0.01$ & $15.87 \pm 0.16$ & $15.91 \pm 0.16$ & $\ldots$ & $\ldots$ \\
\hline$K_{s}^{\mathrm{d}}$ & $12.58 \pm 0.06$ & $13.3 \pm 0.1$ & $\cdots$ & $\cdots$ & $\cdots$ & $8.15 \pm 0.01$ & $15.63 \pm 0.22$ & $15.42 \pm 0.19$ & $\cdots$ & $\ldots$ \\
\hline$W I S E W I^{\mathrm{d}}$ & $11.67 \pm 0.02$ & $13.2 \pm 0.02$ & $15.76 \pm 0.05$ & $15.55 \pm 0.04$ & $17.08 \pm 0.11$ & $7.88 \pm 0.0$ & $14.67 \pm 0.03$ & $14.93 \pm 0.03$ & $14.88 \pm 0.03$ & $15.47 \pm 0.04$ \\
\hline$W I S E W 2^{\mathrm{d}}$ & $10.8 \pm 0.02$ & $13.04 \pm 0.03$ & $14.33 \pm 0.04$ & $14.8 \pm 0.06$ & $16.75 \pm 0.26$ & $7.66 \pm 0.0$ & $13.82 \pm 0.04$ & $14.03 \pm 0.04$ & $14.3 \pm 0.05$ & $14.81 \pm 0.06$ \\
\hline$W I S E W 3^{\mathrm{d}}$ & $7.74 \pm 0.02$ & $9.86 \pm 0.05$ & $11.25 \pm 0.16$ & $12.11 \pm 0.28$ & 12.8 & $3.94 \pm 0.01$ & $11.83 \pm 0.27$ & $10.79 \pm 0.1$ & $11.08 \pm 0.1$ & $11.34 \pm 0.17$ \\
\hline WISE W $4^{\mathrm{d}}$ & $5.46 \pm 0.04$ & $7.7 \pm 0.16$ & $8.23 \pm 0.24$ & $8.9 \pm 0.38$ & 8.75 & $1.83 \pm 0.01$ & $8.76 \pm 0.4$ & $8.72 \pm 0.49$ & $9.11 \pm 0.46$ & $8.66 \pm 0.39$ \\
\hline$M_{r}^{\mathrm{e}}$ & -19.95 & -18.89 & -20.12 & -20.34 & -19.28 & -18.11 & -20.29 & -20.32 & -20.48 & -19.61 \\
\hline Telescope $^{\mathrm{f}}$ & Keck & Keck & Keck & NTT & Keck & SDSS & SDSS & SDSS & NTT & Keck \\
\hline Camera $^{\mathrm{f}}$ & LRIS & LRIS & LRIS & EFOSC2 & DEIMOS & $\cdots$ & $\cdots$ & $\cdots$ & EFOSC2 & LRIS \\
\hline UT date ${ }^{f}$ & 2013 Oct 03 & 2013 Nov 09 & 2015 Dec 05 & 2015 Mar 14 & 2013 Dec 11 & 2003 Apr 25 & 2004 Apr 16 & 2004 Dec 13 & 2015 Mar 14 & 2016 Aug 06 \\
\hline Type $^{g}$ & BLAGN & BLAGN & Galaxy & NLAGN & BLAGN & NLAGN & BLAGN & BLAGN & Galaxy & Galaxy \\
\hline $\log L_{6 \mu \mathrm{m}}(\mathrm{AGN})^{\mathrm{h}}$ & 42.99 & 41.2 & 43.31 & 43.37 & 42.83 & 41.99 & 43.19 & 42.96 & 43.18 & 42.96 \\
\hline$M_{\star}{ }^{\mathrm{i}}$ & 9.8 & 9.0 & 8.9 & 10.0 & 9.4 & 9.1 & 9.9 & 9.8 & 10.0 & 9.5 \\
\hline$M .^{\mathrm{j}}$ & 7.02 & 6.06 & $\cdots$ & $\cdots$ & 7.5 & $\ldots$ & 6.51 & 6.82 & $\cdots$ & $\cdots$ \\
\hline$\lambda_{\text {Eddington }}{ }^{\mathrm{j}}$ & 6.2 & 5.3 & $\cdots$ & $\cdots$ & 6.5 & $\cdots$ & 24.5 & 10.6 & $\cdots$ & $\cdots$ \\
\hline
\end{tabular}

Notes.

${ }^{\mathrm{a}}$ Source name.

b Optical counterpart source position.

${ }^{c}$ Offset between NUSTAR and optical source positions in arcsec.

${ }^{\mathrm{d}}$ Source photometry in its original form, e.g., AB asinh mag for SDSS and Vega for SuperCOSMOS, 2MASS, and WISE bands. See Section 2.2 for details.

e Absolute $r$-band magnitude.

${ }^{\mathrm{f}}$ Observational details of the optical spectroscopy.

g Optical spectroscopic classification. See Section 3.4 for details of the classification.

${ }^{\mathrm{h}}$ Mid-IR luminosity of the AGN component from the best-fitting SED in logarithmic erg s ${ }^{-1}$

${ }^{\mathrm{i}}$ Stellar mass in logarithmic $M_{\odot}$. The typical uncertainty in $M_{\star}$ is 0.3 dex (see Section 3.1)

${ }^{\mathrm{j}}$ Black hole mass in logarithmic $M_{\odot}$ and Eddington ratio in percentage for broad-line AGNs. See Section 5.2 for details. 


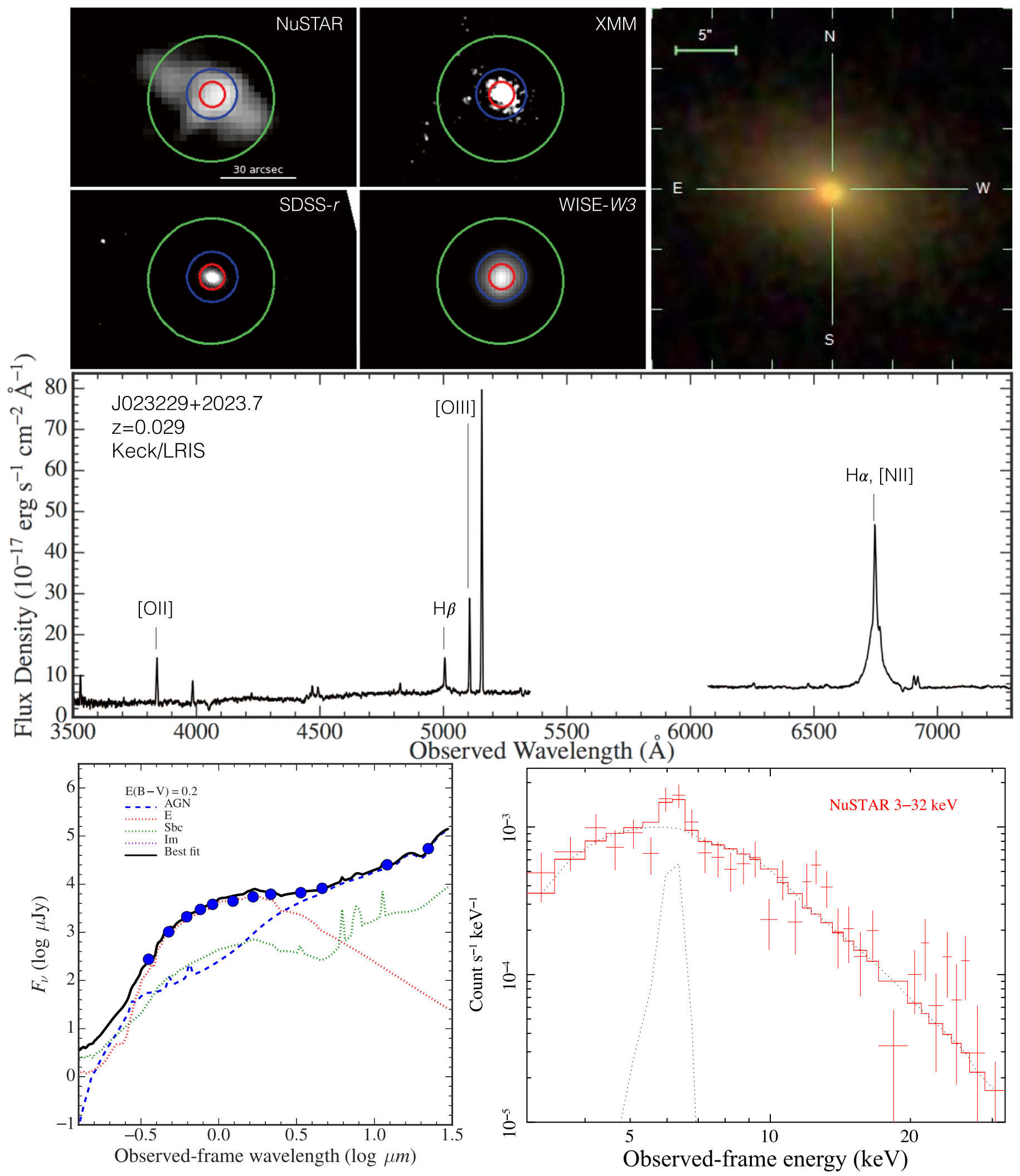

Figure 2. Multiwavelength data of J023229+2023.7. The top left panel shows the location of this object in NuSTAR-FB, XMM-Newton, SDSS $r$, and WISE W3 bands (upper left, upper right, lower left, and lower right, respectively). The green (25" radius), blue (10" radius), and red ( $5^{\prime \prime}$ radius) circles represent the $N u S T A R$, soft $\mathrm{X}$-ray, and optical locations, respectively. The upper right panel shows the false-color image obtained using the SDSS DR12 image cutout tool centered on the associated optical galaxy. The middle panel shows the observed optical spectrum of J023229+2023.7. The multiwavelength photometry and best-fit SED are shown in the bottom left panel. The NUSTAR X-ray spectrum and the best-fit model are shown in the bottom right panel. See Appendix A for a brief summary of this object. The complete figure set (10 images) for the entire NUSTAR low-mass AGN sample is available in the online journal.

(The complete figure set (10 images) is available.) 
simulated spectrum. The resulting goodness of fit is defined as the fraction of simulated spectra with a K-S statistic smaller than that between the best-fit model and data. We consider the fit to be acceptable for sources with goodness $<50 \%$. Depending on the goodness of fit and inspection of the residuals of the basic model, some of the objects require an additional iron $\mathrm{K} \alpha$ line component or an additional diffuse plasma component to achieve an acceptable fit.

The X-ray spectral analysis procedures for each object are presented in Appendix A. For the objects with sufficient X-ray photon counts, we calculate the following X-ray spectral properties using the best-fit model: intrinsic $N_{\mathrm{H}}$ value, X-ray luminosity (not corrected for absorption) measured in the restframe $2-10 \mathrm{keV}$ band $\left(L_{2-10 \mathrm{keV}}\right.$ hereafter $)$, X-ray flux measured in the observed-frame $3-8 \mathrm{keV}$ band, and X-ray power-law photon index $\Gamma$. Uncertainties of fluxes and model parameters are estimated using the $90 \%$ confidence intervals. Three of these seven objects have $N_{\mathrm{H}}>10^{22} \mathrm{~cm}^{-2}$.

For the other three objects in our sample, J120711+3348.5, $\mathrm{J} 134934-3025.5$, and $\mathrm{J} 223654+3423.5$, there are not enough $\mathrm{X}$-ray counts for spectral fitting. We estimate their $3-8 \mathrm{keV} \mathrm{X}$ ray fluxes and $L_{2-10 \mathrm{keV}}$ values from their count rates in the corresponding energy ranges using PIMMS with a photon index of $\Gamma=1.8$ and a Galactic absorption column density. The flux uncertainties for these three objects were obtained using uncertainties of the photon counts estimated with the Gehrels (1986) method. To constrain the basic X-ray spectral properties of these three objects, we estimate their hardness ratios, $(H-S) /(H+S)$, where $H$ and $S$ represent hard-band and soft-band counts, respectively. For the Swift/XRT data of $\mathrm{J} 120711+3348.5$, we measure its $S$ and $H$ at $0.3-2 \mathrm{keV}$ and $2-10 \mathrm{keV}$, respectively. For the XMM-Newton data of J134934-3025.5, we measure its $S$ and $H$ at $0.5-2 \mathrm{keV}$ and $2-10 \mathrm{keV}$, respectively. For the Chandra data of J223654 +3423.5 , we measure its $S$ and $H$ at $0.5-2 \mathrm{keV}$ and $2-8 \mathrm{keV}$, respectively. The hardness ratios and the associated uncertainties are then estimated using BEHR (Park et al. 2006). We also use PIMMS to calculate the corresponding $N_{\mathrm{H}}$ values using the hardness ratios assuming a $\Gamma=1.8$ power-law spectrum.

We list the details of ancillary X-ray observations, X-ray spectral-fitting parameters, and the rest-frame $L_{2-10 \mathrm{keV}}$ (observed and absorption corrected) in Table 4. The angular offsets between the optical positions and the soft X-ray positions are also listed for reference. For objects for which we can do spectral analysis, the rest-frame $L_{10-40 \mathrm{keV}}$ values (both observed and absorption corrected) are also calculated based on the best-fit models and are present in Table 2. Notably, six of the low-mass AGNs (J023229+2023.7, J032459-0256.2, J065318+7424.8, J101609-3329.6, $\mathrm{J} 115851+4243.2$, and $\mathrm{J} 223654+3423.5$ ) in our sample have multiple ancillary X-ray observations. However, these additional observations have limited spatial resolution and/or small photon counts, which prevent us from further assessing their $\mathrm{X}$-ray properties. The details of these additional observations are presented in Appendix B and the second half of Table 4.

\subsection{Optical Spectroscopic Observations and Analysis}

Of the 10 low-mass galaxies, three have existing SDSS spectra. For the other seven galaxies, optical spectroscopic follow-up observations were obtained using either Keck LRIS, Palomar DBSP, or NTT EFOSC2 as part of the spectroscopic follow-up program for the NuSTAR serendipitous survey (L17).
Details of the spectroscopic observations of our sample are listed in Table 3, and the optical spectrum for each object is presented as part of the figure set in Figure 2.

In our sample, only seven of the 10 objects have high-S/N spectra with strong emission lines. We analyze the optical spectra of these seven objects with customized software similar to that used in Reines et al. (2013), which removes the hostgalaxy contribution and models the AGN emission-line profiles. Important features in the optical spectrum that indicate the presence of an accreting $\mathrm{mBH}$ are $\mathrm{H} \alpha$ and $\mathrm{H} \beta$ emission lines that are significantly broadened since they arise from dense gas orbiting an $\mathrm{mBH}$, though these broad Balmer lines are often blended with other narrow emission lines at similar wavelengths. Our analysis method models the blended broad Balmer lines and other high-ionization narrow-line components, yielding accurate emission-line width and flux measurements for the broad Balmer lines and other narrow highionization lines. The details of the model-fitting method are described in Section 3 of Reines et al. (2013). We find that five of the seven objects with high-S/N spectra have a robust broad $\mathrm{H} \alpha$ component with a line width broader than $500 \mathrm{~km} \mathrm{~s}^{-1}$ that is indicative of accretion onto an $\mathrm{mBH}$ (Reines et al. 2013). We show the results of our broad $\mathrm{H} \alpha$ emission line measurements in Figure 3. For completeness, we also show the close-up spectra for sources without broad $\mathrm{H} \alpha$ lines in Figure 11 of Appendix A.

We present the distribution of narrow emission line ratios of [O III] $\lambda 5007 / \mathrm{H} \beta$ versus [N II] $\lambda 6583 / \mathrm{H} \alpha$ (i.e., the "BPT diagram"; Baldwin et al. 1981) in Figure 4, as a diagnostic of the nature of the optical spectra. We find that all seven lowmass AGNs with high-quality optical spectra have emissionline ratios above the Kauffmann et al. (2003) empirical curve separating AGNs from star-forming galaxies and LINERs in the BPT diagram, and six of these seven AGNs also have emission-line ratios above the Kewley et al. (2006) "maximum starburst" curve. This indicates that the emission lines in these objects are powered by accretion onto massive BHs.

\section{The AGN Nature of the Low-mass Galaxies with NuSTAR Detections}

\subsection{The Optical and X-Ray AGN Diagnostics}

One of the challenges in confirming the presence of an AGN in low-mass galaxies is that the AGN emission is often diluted by stellar processes. This is particularly true for objects that are less luminous in the X-ray band (i.e., $L_{2-10 \mathrm{keV}}<10^{42} \mathrm{erg} \mathrm{s}^{-1}$ ) and objects without the telltale high-excitation emission lines in the optical spectra.

To verify that the $10 \mathrm{NUSTAR}$-selected low-mass galaxies are indeed powered by accretion onto an $\mathrm{mBH}$, we consider the following diagnostics:

1. The empirical $L_{2-10 \mathrm{keV}}>10^{42} \mathrm{erg} \mathrm{s}^{-1}$ criterion, which generally distinguishes $\mathrm{X}$-ray emission powered by AGNs from that powered by X-ray binaries (see Section 3.3 and Appendix A for the details of derivation of $L_{2-10 \mathrm{keV}}$ for each object).

2. The optical emission line ratio diagnostics (the BPT diagram; Figure 4). We consider the Kauffmann et al. (2003) and Kewley et al. (2006) curves that separate AGNs from star-forming galaxies and LINERs.

3. The presence of significantly broadened $\mathrm{H} \alpha$ line emission $\left(\mathrm{FWHM}>500 \mathrm{~km} \mathrm{~s}^{-1}\right.$ ), which indicates the 
Table 4

Additional X-Ray Observations and X-Ray Spectral Properties

\begin{tabular}{|c|c|c|c|c|c|c|c|c|c|c|c|c|c|}
\hline $\begin{array}{l}\text { Source Name } \\
\text { (1) }\end{array}$ & $\begin{array}{l}\text { Observatory } \\
\text { (Soft X-ray) } \\
\text { (2) }\end{array}$ & $\begin{array}{l}\text { ObsID } \\
\text { (3) }\end{array}$ & $\begin{array}{l}\text { R.A. } \\
(\text { J2000) } \\
(4)\end{array}$ & $\begin{array}{l}\text { Decl. } \\
(\text { J2000) } \\
(5)\end{array}$ & $\begin{array}{l}\text { Offset } \\
\text { (arcsec) } \\
(6)\end{array}$ & $\begin{array}{c}\text { Exposure } \\
\text { Time } \\
(\mathrm{ks}) \\
(7)\end{array}$ & $\begin{array}{c}\text { Flux } \\
(3-8 \mathrm{keV}) \\
(8)\end{array}$ & $\begin{array}{l}\text { Energy } \\
\text { Range } \\
(\mathrm{keV}) \\
(9)\end{array}$ & $\begin{array}{l}\Gamma \\
(10)\end{array}$ & $\begin{array}{c}\log N_{\mathrm{H}}^{\text {Galactic }} \\
\left(\mathrm{cm}^{-2}\right) \\
(11)\end{array}$ & $\begin{array}{l}\log N_{\mathrm{H}} \\
\left(\mathrm{cm}^{-2}\right) \\
(12)\end{array}$ & $\begin{array}{c}\text { Goodness } \\
(\%) \\
(13)\end{array}$ & $\begin{array}{c}\log L_{2-10 \mathrm{keV}} \\
\left(\mathrm{erg} \mathrm{s}^{-1}\right) \\
(14)\end{array}$ \\
\hline J023229+2023.7 & NUSTAR ${ }^{\mathrm{a}}$ & $\ldots$ & $\ldots$ & $\ldots$ & $\ldots$ & 37.5 & $70.47_{-9.52}^{+9.70}$ & $3-20$ & 1.8 & 20.38 & $23.11_{-0.28}^{+0.21}$ & 32.00 & $42.25(42.56)$ \\
\hline J032459-0256.2 & $\begin{array}{c}\text { Swift } / \text { XRT }+ \\
\text { NuSTAR }\end{array}$ & 0405240201 & 51.24934 & -02.93677 & 1.38 & 16.4 & $36.5_{-5.2}^{+6.1}$ & $0.5-32$ & $1.94_{-0.15}^{+0.34}$ & 20.73 & $<21.53$ & 21.40 & $41.52(41.53)$ \\
\hline J065318+7424.8 & $\begin{array}{c}\text { Chandra }+ \\
\text { NuSTAR }\end{array}$ & 10324 & 103.33762 & 74.417292 & 0.16 & 74.1 & $2.6_{-0.2}^{+0.2}$ & $0.5-32$ & $1.88_{-0.27}^{+0.29}$ & 20.64 & $21.32_{-0.25}^{+0.27}$ & 16.24 & $42.52(42.54)$ \\
\hline J101609-3329.6 & $\begin{array}{r}\text { XMM-Newton } \\
+ \text { NuSTAR }\end{array}$ & 0203890101 & 154.00490 & -33.49289 & 0.24 & 71.5 & $6.7_{-0.7}^{+0.7}$ & $2-24$ & $1.80_{-0.41}^{+0.42}$ & 20.98 & $22.79_{-0.25}^{+0.17}$ & 20.40 & $43.13(43.27)$ \\
\hline $\mathrm{J} 103410+6006.7$ & $X M M$-Newton & 0306050701 & 158.54270 & 60.11219 & 0.53 & 8.8 & $3.91_{-0.7}^{+1.0}$ & $0.5-12$ & $1.67_{-0.17}^{+0.18}$ & 20.20 & $<20.95$ & 17.40 & $43.06(43.06)$ \\
\hline $\mathrm{J} 115851+4243.2$ & Chandra & 17006 & 179.71799 & 42.722333 & 1.2 & 29.6 & $1.5_{-0.3}^{+0.5}$ & $1.0-8$ & 1.8 & 20.17 & $23.06_{-2.5}^{+0.35}$ & 0.10 & $38.43(38.73)$ \\
\hline $\mathrm{J} 120711+3348.5$ & Swift/XRT & 37315 & 181.79622 & 33.812419 & 3.6 & 11.1 & $6.3 \pm 1.8$ & $0.2-10$ & 1.8 & 20.07 & $<20.07^{\mathrm{b}}$ & $\ldots$ & $42.65\left(42.65^{\mathrm{b}}\right)$ \\
\hline J121358+2936.1 & Chandra & 14042 & 183.497154 & 29.60475 & 0.7 & 5.0 & $4.43_{-1.4}^{+1.5}$ & $0.5-8$ & $2.56_{-1.25}^{+2.12}$ & 20.34 & $21.72_{-1.7}^{+0.4}$ & 0.01 & $42.56(42.60)$ \\
\hline J134934-3025.5 & XMM-Newton & 0101040401 & 207.38800 & -30.42731 & 2.0 & 9.2 & $2.3 \pm 0.5$ & $0.2-12$ & 1.8 & 20.93 & $22.0_{-0.7}^{+0.5} \mathrm{~b}$ & $\ldots$ & $42.46\left(42.50^{b}\right)$ \\
\hline $\mathrm{J} 223654+3423.5$ & Chandra & 17570 & 339.22414 & 34.392484 & 0.7 & 9.9 & $0.4 \pm 0.17$ & $0.5-7$ & 1.8 & 21.05 & $22.0_{-0.6}^{+0.2} \mathrm{~b}$ & $\ldots$ & $41.59\left(41.63^{\mathrm{b}}\right)$ \\
\hline
\end{tabular}

Additional Soft

X-ray

Observations $^{\mathrm{c}}$

$\mathrm{J} 023229+2023.7 \mathrm{a}$

$\mathrm{J} 023229+2023.7 \mathrm{~b}$

J032459-0256.2a

J065318+7424.8a

$\mathrm{J} 065318+7424.8 \mathrm{~b}$

J101609-3329.6a

$\mathrm{J} 115851+4243.2 \mathrm{a}$

J223654+3423.5a

$\mathrm{J} 223654+3423.5 \mathrm{~b}$

$\begin{array}{lrcrl}\text { XMM-Newton } & 0604210201 & 38.11999 & 20.39719 & 0.07 \\ \text { XMM-Newton } & 0604210301 & 38.11995 & 20.39723 & 0.25 \\ \text { XMM-Newton } & 0405240201 & 51.24934 & -02.93677 & 0.48 \\ \text { XMM-Newton } & 0061540101 & 103.33733 & 74.41740 & 0.64 \\ \text { XMM-Newton } & 0144230101 & 103.33738 & 74.41739 & 0.58 \\ \text { Chandra } & 0203890101 & 154.00490 & -33.49289 & 0.25 \\ \text { XMM-Newton } & 0744040301 & \ldots & \ldots & 3.84 \\ \text { Chandra } & 17569 & 339.224 & 34.393341 & 3.05 \\ \text { Chandra } & 17571 & 339.22437 & 34.393573 & 3.69\end{array}$

$\begin{array}{lrcll}0.07 & 8.7 & 43.4 & 0.2-12 & 1.8 \\ 0.25 & 10.0 & 27.2 & 0.2-12 & 1.8 \\ 0.48 & 11.8 & 10.2 & 0.2-12 & 1.8 \\ 0.64 & 7.1 & 5.1 & 0.2-12 & 1.8 \\ 0.58 & 29.2 & 2.0 & 0.2-12 & 1.8 \\ 0.25 & 7.2 & 7.3 & 0.5-8 & 1.8 \\ 3.84 & 18.0 & 2.68 & 0.2-12 & 1.8 \\ 3.05 & 9.9 & 0.4 & 0.5-8 & 1.8 \\ 3.69 & 9.9 & 0.2 & 0.5-8 & 1.8\end{array}$

$\begin{array}{ll}1.8 & 20.38 \\ 1.8 & 20.38 \\ 1.8 & 20.73 \\ 1.8 & 20.64 \\ 1.8 & 20.64 \\ 1.8 & 20.98 \\ 1.8 & 20.17 \\ 1.8 & 21.05 \\ 1.8 & 21.05\end{array}$

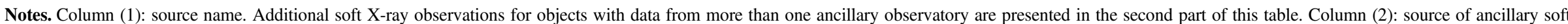

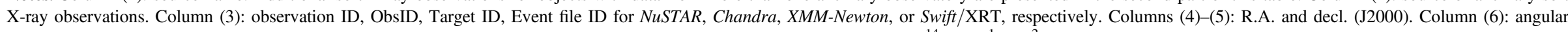

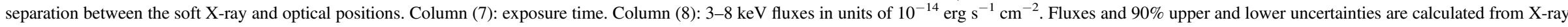

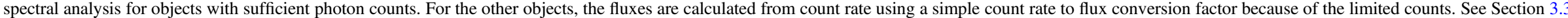

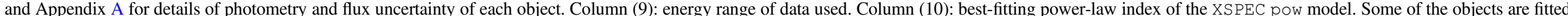

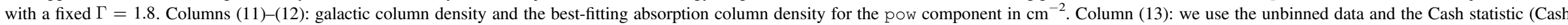

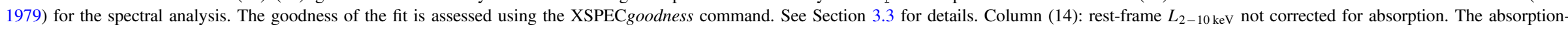

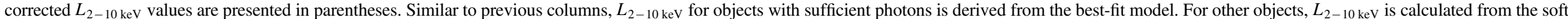

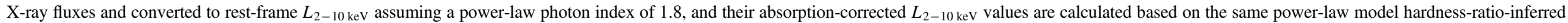
$N_{\mathrm{H}}$ values.

a Due to the XMM-Newton position of J023229+2023.7 being located near the chip gap, we estimate its soft X-ray properties using NuSTAR data.

${ }^{\mathrm{b}} N_{\mathrm{H}}$ is estimated based on the hardness ratio assuming a $\Gamma=1.8 \mathrm{X}$-ray spectrum using PIMMS

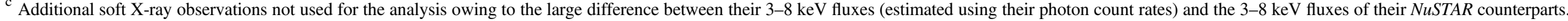



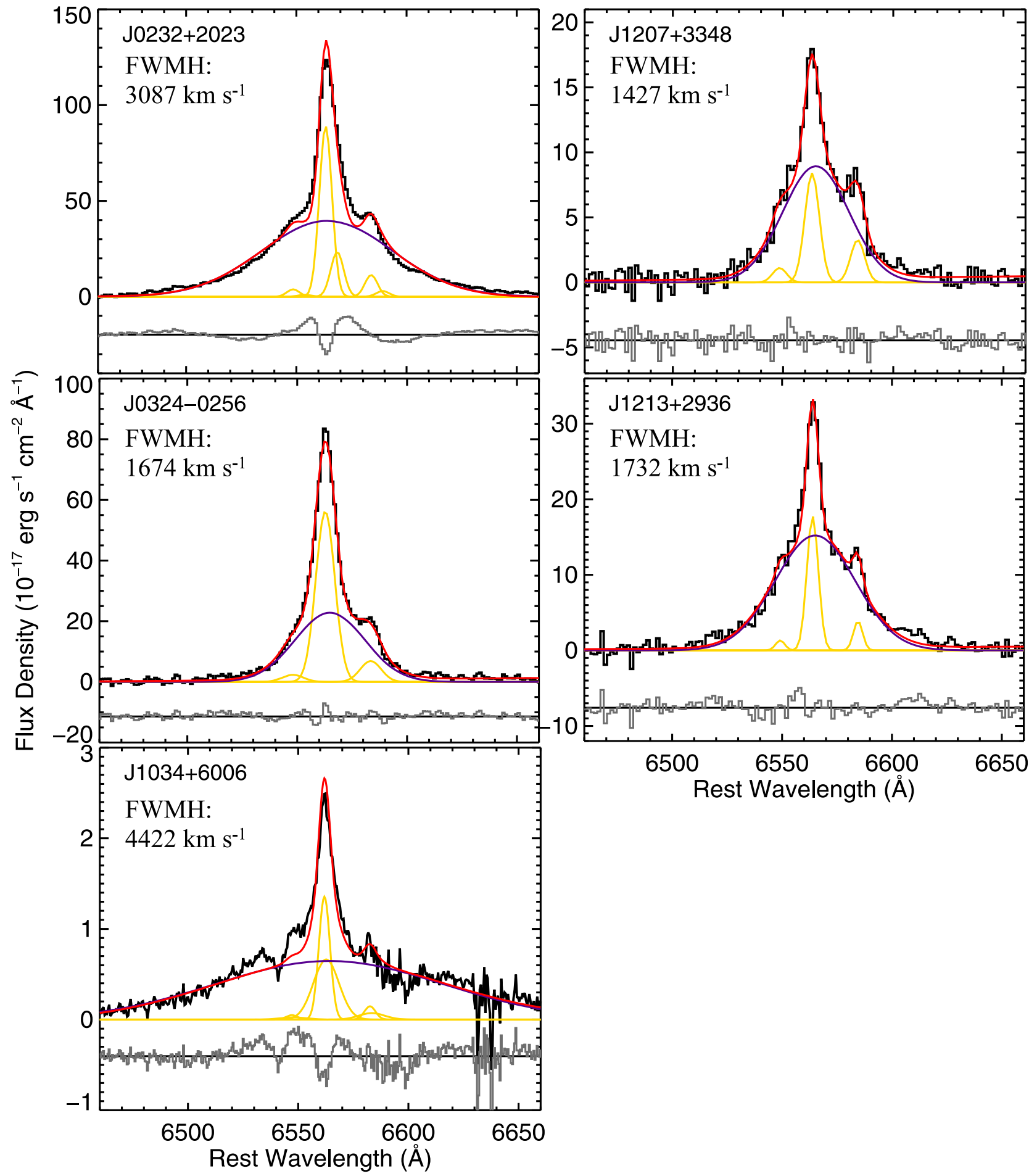

Figure 3. Continuum- and absorption-line-subtracted spectra showing the rest-frame broad $\mathrm{H} \alpha$ lines. For each object, the best-fitting model is shown in red. Individual Gaussian components of narrow lines are shown in yellow. The broad $\mathrm{H} \alpha$ component is plotted in dark blue. Residuals are plotted in gray with a vertical offset for clarity.

presence of dense gas being accreted onto an $\mathrm{mBH}$ (see Figure 3).

For the 10 galaxies in our sample, eight of them have $L_{2-10 \mathrm{keV}}>10^{42} \mathrm{erg} \mathrm{s}^{-1}$ that can be securely attributed to an $\mathrm{X}$-ray AGN. As for the optical emission line diagnostics, all seven objects in our sample with high-S/N optical spectra reside in the AGN region defined using the Kauffmann et al.
(2003) curves. Of these seven objects, all except one, J032459-0256.2, are also above the Kewley et al. (2006) "maximum starburst" curve. Although the emission-line ratios of J032459-0256.2 do not exceed the Kewley et al. (2006) curve, its optical spectrum shows a significant broad $\mathrm{H} \alpha$ component (FWHM $=1674 \mathrm{~km} \mathrm{~s}^{-1}$ ) that is likely to be powered by accretion onto an $\mathrm{mBH}$. Therefore, we consider J032459-0256.2 to be an optical AGN as well. 


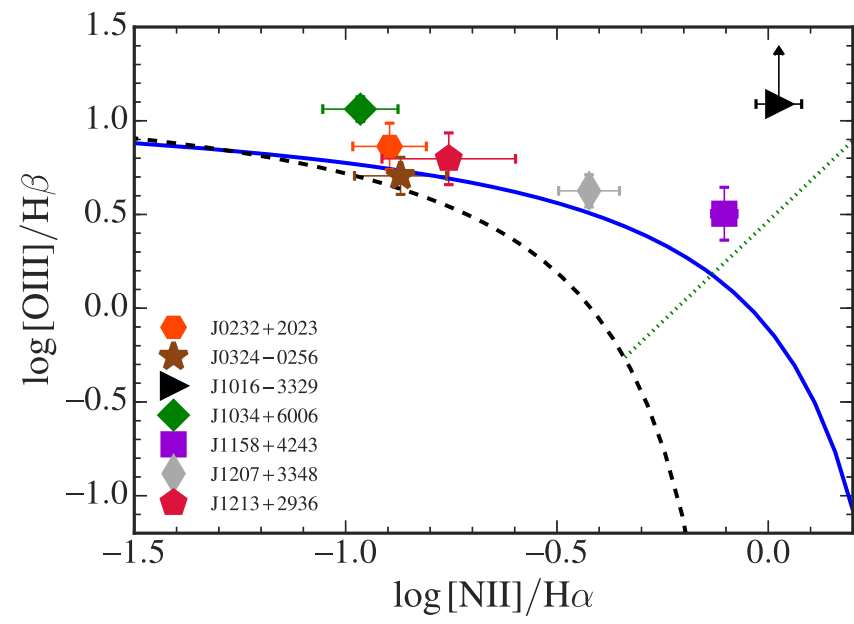

Figure 4. Emission-line ratios (BPT diagram) for the NuSTAR low-mass AGNs with high-S/N optical spectra. All lie above the Kauffmann et al. (2003) empirical curve (dashed curve that defines the "star-forming region" and the dotted line that separates LINERs from AGNs) for selecting galaxies with significant AGN contributions to the emission lines. The Kewley et al. (2006) "maximum starburst" line (solid curve) is also shown.

Table 5

AGN Identification Criteria

\begin{tabular}{lllll}
\hline \hline $\begin{array}{l}\text { Source Name } \\
(1)\end{array}$ & $\begin{array}{l}L_{2-10 \mathrm{keV}} \\
(2)\end{array}$ & $\begin{array}{l}\text { Broad H } \alpha \\
(3)\end{array}$ & $\begin{array}{l}\text { BPT Diagram } \\
(4)\end{array}$ & $\begin{array}{l}\text { WISE Color } \\
(5)\end{array}$ \\
\hline $\mathrm{J} 023229+2023.7$ & Yes & Yes & Yes & Yes \\
$\mathrm{J} 032459-0256.2$ & No & Yes & Yes $^{\mathrm{a}}$ & No \\
$\mathrm{J} 065318+7424.8$ & Yes & No & No & Yes \\
$\mathrm{J} 101609-3329.6$ & Yes & No & Yes & No \\
$\mathrm{J} 103410+6006.7$ & Yes & Yes & Yes & No \\
$\mathrm{J} 115851+4243.2$ & No & No & Yes & No \\
$\mathrm{J} 120711+3348.5$ & Yes & Yes & Yes & Yes \\
$\mathrm{J} 121358+2936.1$ & Yes & Yes & Yes & Yes \\
$\mathrm{J} 134934-3025.5$ & Yes & No & No & No \\
J223654+3423.5 & Yes & No & No & No \\
\hline
\end{tabular}

Notes. The summary table for the results from Section 4.1. Column (1): source name. Column (2): the $L_{2-10 \mathrm{keV}}>10^{42} \mathrm{erg} \mathrm{s}^{-1}$ criterion. Column (3): the presence of a broad $\mathrm{H} \alpha$ line; see Figure 3. Column (4): the BPT emission-line ratio diagnostics; see Figure 4. Column (5): the Stern et al. (2012) WISE colorselection criterion, $W 1-W 2>0.8$. See Section 5.1 for details.

a The emission-line ratios of this object do not exceed the "maximum starburst curve" defined by Kewley et al. (2006), but still reside in the AGN region defined by the Kauffmann et al. (2003) curves on the BPT diagram.

${ }^{\mathrm{b}}$ The $L_{2-10 \mathrm{keV}}$ derived based on the Chandra observations does not exceed $10^{42} \mathrm{erg} \mathrm{s}^{-1}$, but the NuSTAR-SB flux does correspond to a soft X-ray luminosity satisfying $L_{2-10 \mathrm{keV}}>10^{42} \mathrm{erg} \mathrm{s}^{-1}$.

We list the results of these diagnostics in Table 5. While we do not use the mid-IR colors to determine the presence of an AGN for the low-mass galaxies in our sample, we also list whether our sample objects satisfy the Stern et al. (2012) WISE color-selection criterion, $W 1-W 2>0.8$, in Table 5. A detailed discussion of the validity of using WISE color to identify AGNs in low-mass galaxies is given in Section 5.1.

For our sample, there are a total of five objects that satisfy both the X-ray luminosity and optical emission line diagnostics; we consider these seven objects to be "multiwavelength" AGNs (see the previous subsection and Table 5). For the other five objects in our sample, three of them (J065318+7424.8, J134934-3025.5, and J223654+3423.5) have $L_{2-10 \mathrm{kev}}>10^{42} \mathrm{erg} \mathrm{s}^{-1}$ in at least one epoch of X-ray observations but have no apparent optical emission lines that could be used for emission-line diagnostics. These "optically dull" X-ray AGNs are discussed in more detail in Section 4.2.

Besides the five multiwavelength AGNs and the three optically dull X-ray AGNs, there are two X-ray faint sources in our sample that are classified as an AGN at optical wavelengths: J032459-0256.2 and J115851+4243.2. Their $\mathrm{X}$-ray luminosities are fainter than the empirical $10^{42} \mathrm{erg} \mathrm{s}^{-1}$ threshold for identifying typical X-ray AGNs. For J032459-0256.2, the soft X-ray luminosity, $L_{2-10 \mathrm{keV}}=$ $3.3 \times 10^{41} \mathrm{erg} \mathrm{s}^{-1}$, is derived by jointly fitting the available NuSTAR and Swift/XRT data. We find that the X-ray spectrum of J032459-0256.2 is consistent with an absorbed power-law with $\Gamma=1.9_{-0.2}^{+0.3}$ and intrinsic $N_{\mathrm{H}}<3.4 \times 10^{21} \mathrm{~cm}^{-2}$. Although its $2-10 \mathrm{keV}$ X-ray luminosity does not exceed the $10^{42} \mathrm{erg} \mathrm{s}^{-1}$ threshold, the optical spectrum exhibits strong broad and narrow high-ionization emission lines powered by an AGN. The BH mass of this object is $\log M_{\bullet} / M_{\odot}=6.06$ based on its broad $\mathrm{H}_{\alpha}$ emission. With the clear optical AGN signatures and the low $M_{\text {. }}$, we consider the $\mathrm{X}$-ray emission from J032459-0256.2 to be indeed powered by accretion onto $\mathrm{mBH}$, and J032459-0256.2 is also a "multiwavelength" AGN.

For J115851+4243.2, the soft X-ray luminosity is $L_{2-10 \mathrm{keV}}=2.7 \times 10^{38} \mathrm{erg} \mathrm{s}^{-1}$. In this luminosity range, it is also possible for the X-ray emission to be powered by X-ray binaries or even a single ULX. However, the optical emission line ratios of J115851+4243.2 suggest the presence of an underlying AGN. The high spatial resolution Chandra image also reveals that the position of $>2 \mathrm{keV}$ X-ray emission coincides with the SDSS fiber location with $<0$ "' 3 separation (see Table 3). Considering the absolute astrometric uncertainties of Chandra and SDSS ( $<1$ ". 1 and $<0$ " 1 , respectively), the physical separation between the hard X-ray source and the SDSS fiber location is less than $\approx 50$ pc. In Section 4.3 we explore further the nature of $\mathrm{J} 115851+4243.2$ and show that the weak X-ray emission of J115851+4243.2 might be due to the presence of large amounts of obscuring material.

\subsection{Optically Dull X-Ray AGNs}

In our sample, there are three objects with luminous X-ray emission but no obvious AGN-powered emission lines. In detail, the optical spectrum of J134934-3025.5 is consistent with that of a quiescent galaxy with weak emission lines (see online Figure set 2.9). For J223654+3423.5, while its optical spectrum shows strong $\mathrm{H} \alpha$ and [N II] emission lines, there is no apparent [O III] emission. For J065318+7424.8, the optical spectrum has limited $\mathrm{S} / \mathrm{N}$ but no immediately visible evidence indicative of AGN-powered high-ionization lines (see online Figure set 2.3).

There have been extensive studies of the population of objects with bright X-ray nuclei and weak or no optical emission lines (e.g., Elvis et al. 1981; Comastri et al. 2002; Rigby et al. 2005; Civano et al. 2007; Trump et al. 2011). The lack of AGN-powered optical emission lines in these X-raybright objects has often been attributed to a combination of the following reasons: (i) host-galaxy dilution due to the large optical fiber/slit radius (Moran et al. 2002), (ii) parsec-scale obscuration, (iii) a scenario in which the SMBH is accreting at a low Eddington rate via a radiatively inefficient accretion flow (RIAF; e.g., Yuan \& Narayan 2004), or (iv) the radiation from 
the recently triggered $\mathrm{SMBH}$ accretion has yet to reach the narrow emission line regions (e.g., Schawinski et al. 2015).

Considering the luminous X-ray emission and low stellar mass of the objects described in the previous paragraph, it is unlikely that their "optical dullness" is due to underlying RIAFpowered AGNs. For instance, the Eddington ratio for $\mathrm{J} 134934-3025.5$, the most massive object in our NuSTAR sample, would be $L / L_{\text {Eddington }} \sim 0.2$ if we derive $M$. using the Reines \& Volonteri (2015) $M_{\bullet}-M_{\star}$ relation for local AGNs. This is much higher than the threshold for an RIAF, $L_{\text {bolometric }} / L_{\text {Eddington }} \lesssim 0.01$ (e.g., Begelman \& Rees 1984; Narayan et al. 1995; Yuan \& Narayan 2004). On the other hand, the NUSTAR band ratios of the three objects without apparent AGN-powered emission lines are $\gtrsim 0.9$. These values are consistent with flat power-law X-ray spectra with $\Gamma \sim 1.2$, suggesting that the AGNs in these objects might be obscured. However, the uncertainty of the NUSTAR band ratios is substantial for the low-luminosity objects in our sample, due to high background counts caused by the angular proximity to the bright primary science targets. The current soft X-ray data for these three objects are also limited by small numbers of photons. Therefore, additional X-ray and optical spectroscopic observations are required to determine the cause of the lack of optical emission lines in these three objects.

\subsection{Identifying Heavily Obscured AGNs in Low-mass Galaxies with NuSTAR}

The ratio between observed $L_{10-40 \mathrm{keV}}$ and $L_{2-10 \mathrm{keV}}$ has been considered as a basic indicator of obscuration since hard X-rays are relatively insensitive to the presence of obscuring material in comparison with the soft X-rays. For the low-mass AGNs in our sample, we study their $L_{10-40 \mathrm{keV}} / L_{2-10 \mathrm{keV}}$ ratios to investigate how many of them are heavily obscured.

While $L_{10-40 \mathrm{keV}}$ is much less affected by absorption than $L_{2-10 \mathrm{keV}}$, it can still be significantly suppressed when the obscuring column reaches $N_{\mathrm{H}} \gtrsim 10^{24} \mathrm{~cm}^{-2}$ (e.g., Lansbury et al. 2014, 2015, 2016; Stern et al. 2014; LaMassa et al. 2016). Therefore, we also utilize the mid-IR luminosities measured from SED decomposition for our sample. AGN mid-IR and soft $\mathrm{X}$-ray (e.g., rest-frame $2-10 \mathrm{keV}$ ) emission has been found to have an almost 1-to-1 correlation in unobscured Seyfert AGNs (e.g., Fiore et al. 2009; Gandhi et al. 2009; Asmus et al. 2015; Mateos et al. 2015; Stern 2015; Chen et al. 2017) similar to $L_{10-40 \mathrm{keV}}$ and $L_{2-10 \mathrm{keV}}$. Therefore, the ratio between $L_{\mathrm{MIR}}$ and $L_{2-10 \mathrm{keV}}$ has been widely adopted as an indicator of obscuration toward the nucleus (Alexander et al. 2008; Del Moro et al. 2015).

To identify objects with extreme obscuration, we investigate the distributions of the two different luminosity ratios mentioned above. We calculate the $6 \mu \mathrm{m}$ monochromatic luminosity $\left(\nu L_{\nu}\right.$ measured at rest-frame $6 \mu \mathrm{m}, L_{6 \mu \mathrm{m}}$ hereafter) for the AGN component of the best-fit SEDs described in Section 3.1. The two luminosity ratios, $L_{10-40 \mathrm{keV}} / L_{2-10 \mathrm{keV}}$ and $L_{6 \mu \mathrm{m}} / L_{2-10 \mathrm{keV}}$, are shown in Figure 5.

Overall, $L_{10-40 \mathrm{keV}} / L_{2-10 \mathrm{keV}}$ and $L_{6 \mu \mathrm{m}} / L_{2-10 \mathrm{keV}}$ appear plausibly to follow a simple relation for our $N u S T A R$-selected low-mass AGNs, including objects without distinct AGNpowered emission lines and objects that are heavily obscured at soft X-ray energies. This is not surprising, as objects that are more heavily obscured have a weaker $L_{2-10 \mathrm{keV}}$ relative to $L_{10-40 \mathrm{keV}}$ and $L_{6 \mu \mathrm{m}}$. The median difference between the $L_{\mathrm{MIR}}$ of our sample and that derived from their intrinsic $L_{2-10 \mathrm{keV}}$

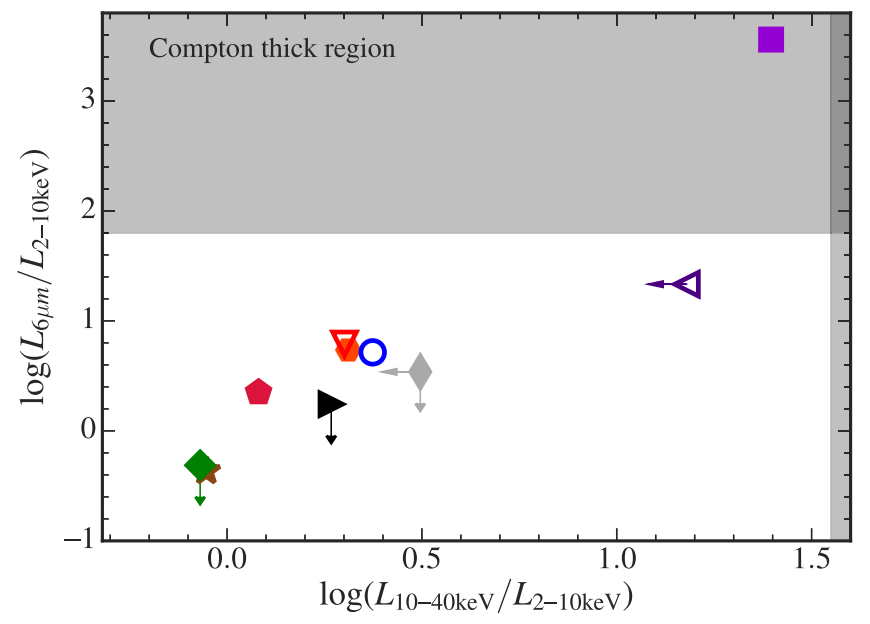

Figure 5. Distributions of two AGN luminosity ratios, $L_{\mathrm{MIR}} / L_{2-10 \mathrm{keV}} \mathrm{vs}$. $L_{10-40 \mathrm{kev}} / L_{2-10 \mathrm{keV}}$. The shaded area is the "Compton-thick region," which is defined by assuming an absorbed power-law X-ray spectrum with $\Gamma=1.8$ and $N_{\mathrm{H}}>1.5 \times 10^{24} \mathrm{~cm}^{-2}$. The more heavily obscured objects occupy the upper right corner of the plot. In particular, J115851+4243.2 (filled purple square) appears to be obscured by Compton-thick column densities (see Section 4.3). Symbols are as in Figure 1.

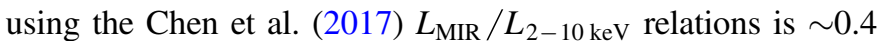
dex, suggesting a non-negligible residual mid-IR host-galaxy contamination due to the large PSF size of WISE. We also caution that the two luminosity ratios shown in Figure 5 have the same denominator $\left(L_{2-10 \mathrm{keV}}\right)$, but the purpose of Figure 5 is not to study the correlation between the intrinsic AGN luminosity ratios, but rather just to identify objects in our sample that might have extreme obscuration. For instance, in the upper right part of Figure 5, there are two objects, J115851 +4243.2 and J223654+3423.5, with $L_{6 \mu \mathrm{m}} / L_{2-10 \mathrm{keV}}>10$.

For $\mathrm{J} 223654+3423.5$, there are not enough X-ray photons to determine the obscuring column density using X-ray spectral modeling. However, the Chandra hardness ratio of J223654 +3423.5 is $\approx 0.1$, which is consistent with that of obscured AGNs, $N_{\mathrm{H}} \approx 10^{22} \mathrm{~cm}^{-2}$ (see Section 3.3 and Table 4).

For J115851+4243.2, the mid-IR luminosity is higher than $L_{2-10 \mathrm{keV}}$ by $3.4 \mathrm{dex}$, and the difference between $L_{10-40 \mathrm{keV}}$ and $L_{2-10 \mathrm{keV}}$ is only $1.4 \mathrm{dex}$. While the large intrinsic column density derived from fitting the Chandra data $\left(N_{\mathrm{H}} \approx 1.2_{-1.0}^{+1.4} \times 10^{23} \mathrm{~cm}^{-2}\right.$; see Appendix A) supports the presence of heavy obscuring material, the 3.4 dex difference between $L_{2-10 \mathrm{keV}}$ and $L_{6 \mu \mathrm{m}}$ requires that $\mathrm{J} 115851+4243.2$ be obscured by Compton-thick material if the intrinsic $L_{2-10 \mathrm{keV}}$ follows the linear $L_{\mathrm{MIR}}$ and $L_{2-10 \mathrm{keV}}$ relations for Seyfert 1 AGNs. Notably, the optical SED of J115851+4243.2 is entirely dominated by the host-galaxy component (see online Figure set 2.6), which also suggests that the AGN is heavily obscured. Furthermore, J115851+4243.2 is extended in all four bands of the WISE images. Therefore, the mid-IR luminosity derived based on the WISE photometry may have significant host-galaxy contamination. Indeed, the mounting evidence for the tight correlation between AGN mid-IR luminosity and $\mathrm{X}$-ray luminosity for local Seyfert galaxies is derived based on high angular resolution $\approx 12 \mu \mathrm{m}$ observations (e.g., Gandhi et al. 2009; Asmus et al. 2015). In these studies, the nuclear mid-IR luminosity and $L_{2-10 \mathrm{keV}}$ are found to have an almost linear correlation. We emphasize that our SED-fitting approach has taken the contribution from host galaxies into account 

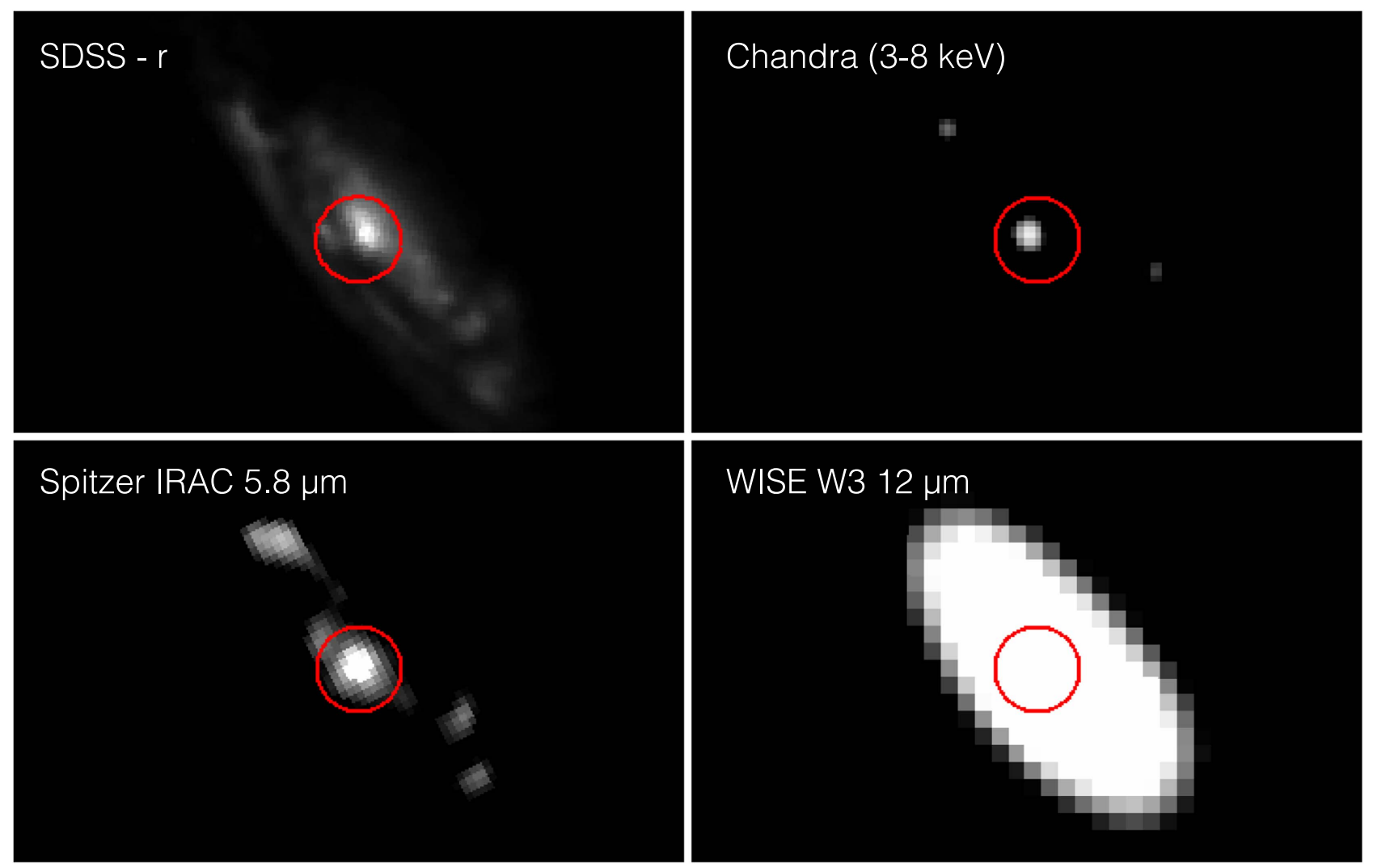

Figure 6. Images of J115851+4243.2 at SDSS $r$ (top left), Chandra 3-8 keV (top right), Spitzer IRAC $5.8 \mu \mathrm{m}$ (bottom left), and WISE W3 (bottom right) bands. The red circle with a $5^{\prime \prime}$ radius defines the region used for aperture photometry at the Spitzer IRAC $5.8 \mu \mathrm{m}$ band. This figure demonstrates that J115851+4243.2 has a powerful nucleus at both mid-IR and 3-8 keV bands, suggesting the presence of an obscured AGN.

when measuring $L_{6 \mu \mathrm{m}}$. For J115851+4243.2, the best-fit SED does imply that the stellar emission remains non-negligible in the $W 1$ and $W 2$ bands (see online Figure set 2.6), but the hostgalaxy contribution rapidly drops at longer wavelengths.

To test whether the SED-decomposed mid-IR AGN luminosity for J115851+4243.2 still suffers from host-galaxy contamination, we obtained the Spitzer Infrared Array Camera (IRAC, Fazio et al. 2004) image for J115851+4243.2 at $5.8 \mu \mathrm{m}$ from the Spitzer Heritage Archive. ${ }^{29}$ The PSF of the IRAC $5.8 \mu \mathrm{m}$ band has a 1". 88 FWHM, which is smaller than the $>6$ " FWHM of WISE PSFs. For comparison, we show the images of J115851+4243.2 at the SDSS $r$ band, Chandra 3-8 keV band, Spitzer IRAC $5.8 \mu \mathrm{m}$ band, and WISE W3 band in Figure 6. In the IRAC $5.8 \mu \mathrm{m}$ image, J115851+4243.2 also appears to host a powerful point source near its center. We measure the IRAC $5.8 \mu \mathrm{m}$ flux for the central source using an aperture of $5^{\prime \prime}$ radius with the MOsaicker and Point source EXtractor (MOPEX; Makovoz \& Marleau 2005). ${ }^{30}$ The measured $5.8 \mu \mathrm{m}$ flux is $(9.8 \pm 1.0) \times 10^{-2} \mathrm{Jy}$, which corresponds to a mid-IR luminosity of $L_{6 \mu \mathrm{m}}=6.0 \times 10^{41}$ erg $\mathrm{s}^{-1}$. This is only $\sim 0.2$ dex lower than the mid-IR luminosity measured based on SED fitting with WISE photometry, $L_{6 \mu \mathrm{m}}=9.7 \times 10^{41} \mathrm{erg} \mathrm{s}^{-1}$, suggesting that the large $(\approx 3.4$ dex $)$ difference between the mid-IR and X-ray luminosities of J115851+4243.2 shown in Figure 5 might indeed be caused by nuclear obscuration.

\footnotetext{
29 http://irsa.ipac.caltech.edu/applications/Spitzer/SHA/

30 http://irsa.ipac.caltech.edu/data/SPITZER/docs/dataanalysistools/tools/ mopex/
}

Since further resolving the nuclear mid-IR emission of $\mathrm{J} 115851+4243.2$ is not possible even with the Spitzer IRAC images, it is informative to consider its optical spectrum (Figure set 2.6). The strong $\mathrm{Ca}$ II and $\mathrm{Na} \mathrm{D}$ absorption lines in the optical spectrum and the red optical color $(u-r \approx 3.1)$ imply that the galaxy is dominated by an old stellar population. The emission-line ratios are also consistent with those of an optical AGN (see Section 3.4). Thus, it is not likely that $\mathrm{J} 115851+4243.2$ is a starburst galaxy powerful enough to generate $L_{6 \mu \mathrm{m}} \approx 10^{42} \mathrm{erg} \mathrm{s}^{-1}$ without an underlying AGN. In fact, if we assume that all the mid-IR emission at W3 is powered by star formation activity, its specific star formation rate estimated using its $8-1000 \mu \mathrm{m}$ infrared luminosity $\left(L_{\mathrm{IR}} \sim 2.8 \times 10_{\odot}^{9 L}\right.$, estimated using the Chary \& Elbaz 2001 star-forming galaxy templates) and $M_{\star}$ would be more than 10 times higher than that of the Milky Way. This is not plausible for a galaxy with a spectrum dominated by an old stellar population. Therefore, the luminous mid-IR emission is more likely to be dominated by an obscured AGN.

It is also interesting that $\mathrm{J} 115851+4243.2$ is tentatively identified as a water megamaser AGN (Darling 2014). Several previous studies have pointed out that AGNs with megamaser emission are likely to be obscured by Compton-thick column densities because the observation of masers requires an edge-on view of the accretion disk (Zhang et al. 2006; Greenhill et al. 2008; Masini et al. 2016). Therefore, the large difference between the $L_{6 \mu \mathrm{m}}$ and $L_{2-10 \mathrm{keV}}$ of $\mathrm{J} 115851+4243.2$ appears to be due to the presence of a Compton-thick obscuring column density. The science target with a $39 \mathrm{ks}$ NuSTAR observation, IC 751 (Ricci et al. 2016), is 9!13 away from J115851+4243.2. 
With the rapid degradation of higher-energy band sensitivity at large off-axis angles, J115851+4243.2 has only been detected at $\approx 2 \sigma$ significance in the $8-24 \mathrm{keV}$ band. For the Chandra data, the best-fit intrinsic column density is $N_{\mathrm{H}}=1.2_{-1.0}^{+1.4} \times 10^{23} \mathrm{~cm}^{-2}$ (see Appendix A), but the $>3 \mathrm{keV}$ photon counts are very limited $(\approx 10$ photons only) and therefore cannot rule out higher obscuring column densities, especially considering that the real X-ray spectrum is likely more complex than our basic modeling (e.g., Baloković et al. 2015). Therefore, a future on-axis observation with a longer exposure time by NUSTAR is required to reveal whether J115851+4243.2 is indeed a Compton-thick AGN hosted by a low-mass galaxy. Also, mid-IR imaging with subarcsecond resolution can provide insight into the origin of the luminous mid-IR emission of J115851+4243.2.

\section{Comparison with Previous Studies}

To evaluate the effectiveness of using NuSTAR to select lowmass AGNs, we compare the properties of our sample with the other relevant AGN samples reported in the literature. We discuss mid-IR colors in Section 5.1, the $M_{\star}-M_{\text {. }}$ relation in Section 5.2, and X-ray properties in Section 5.3.

\subsection{Mid-IR Colors}

Similar to hard X-rays, mid-IR observations are a powerful tool for studying AGN activity that could be enshrouded by intervening dust. Many studies have utilized Spitzer and WISE observations to show that the distinctive red mid-IR color arising from hot dust heated by SMBH accretion can be used as an effective indicator of intrinsically luminous AGN activity (e.g., Lacy et al. 2004; Stern et al. 2005, 2012; Donley et al. 2012; Assef et al. 2013; Mateos et al. 2013). However, AGN identification methods based on red mid-IR colors become more ambiguous for low-luminosity AGNs (e.g., Hainline et al. 2016). In particular, recent studies have found that the vast majority of low-mass galaxies with red mid-IR color do not show any sign of optical emission lines powered by AGNs (Satyapal et al. 2014, 2016; Sartori et al. 2015; Secrest et al. 2015). Several studies have also demonstrated that young, compact starbursts in dwarf galaxies can have mid-IR colors mimicking those of luminous AGNs (e.g., Griffith et al. 2011; Izotov et al. 2014; Hainline et al. 2016). Here we explore whether our hard X-ray-selected low-mass galaxies have mid-IR emission powered by AGN-heated dust to assess the effectiveness of using mid-IR emission to search for obscured AGNs in low-mass galaxies.

We first show the WISE color-color distribution of our lowmass galaxy sample in Figure 7. As mentioned earlier, all the NuSTAR low-mass galaxies have S/Ns higher than 5 in the $W 1$ and $W 2$ bands. There are three objects in our sample with $\mathrm{S} / \mathrm{N}$ $<5$ in the $W 3$ band, and thus we consider their $W 2-W 3$ colors to be upper limits. Only five of the 10 objects in our sample satisfy the Stern et al. (2012) mid-IR AGN selection criteria for luminous AGNs, e.g., $W 1-W 2>0.8$ and $W 2<15$. For our sample, the three least $\mathrm{X}$-ray-luminous sources all have blue mid-IR colors $(W 1-W 2<0.8)$, while the more X-ray-luminous AGNs are more likely to lie above the $W 1-W 2>0.8$ criterion.

By design, the WISE color-selection criterion has limited selection completeness for low-luminosity AGNs, due to more significant host-galaxy dilution (e.g., Eckart et al. 2010; Donley

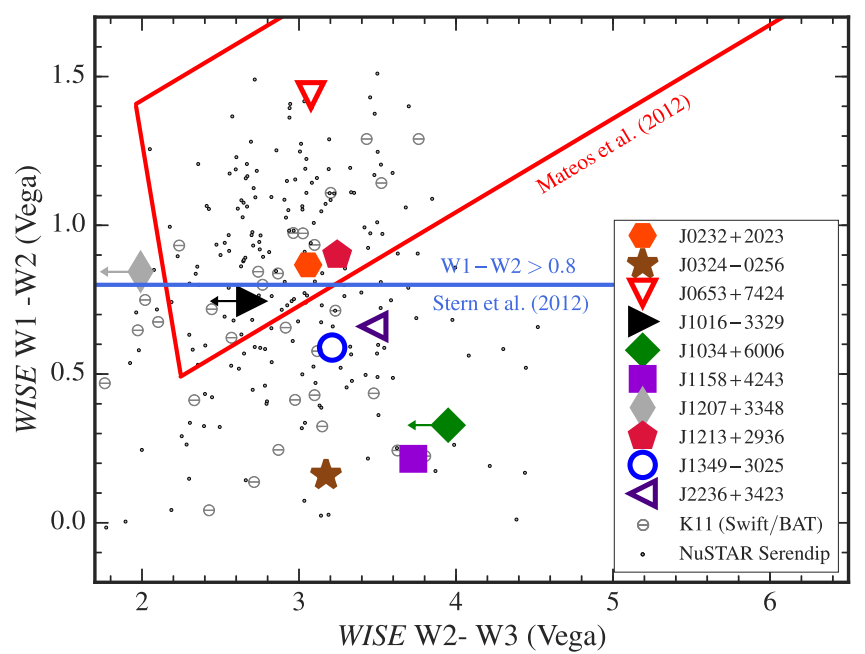

Figure 7. WISE $W 1-W 2$ vs. $W 2-W 3$ distribution for the NUSTAR lowmass AGN sample overplotted on the Mateos et al. (2013; red wedge) and Stern et al. (2012; blue line) mid-IR AGN selection criteria. The entire spectroscopic sample of the NUSTAR serendipitous survey is shown as gray dots. For comparison, the low-mass galaxies selected from the Swift/BAT survey (Koss et al. 2011) are also shown as barred circles (see Section 5.3 for more details of the Swift/BAT sample). Large symbols are as in Figure 1.

et al. 2012). The fraction of AGNs in our sample with $W 1-W 2>0.8$ is also consistent with the $20 \%-40 \%$ mid-IR selection completeness for low-luminosity AGNs in the sample selected using 4.5-10 keV XMM-Newton detection (Mateos et al. 2013) and the complete NUSTAR serendipitous-survey sample (L17). Due to the low completeness of the $W 1-W 2>0.8$ selection criterion, only one out of the three objects without apparent AGN-powered emission lines has $W 1-W 2>0.8$ (namely, J065318+7424.8; see Section 3.4).

Also, two of the most heavily X-ray-obscured AGNs (J101609-3329.6 and J115851+4243.2; see Table 4 and Figure 7) do not meet the $W 1-W 2>0.8$ color-selection criterion. This suggests that mid-IR color-selection methods are not effective in identifying optically normal AGNs hosted by low-mass galaxies. On the other hand, we find that all seven objects in our sample with $\mathrm{S} / \mathrm{N}>5$ in the $W 3$ band have red $W 2-W 3$ colors. Furthermore, the broadband SEDs of these objects show that the mid-IR emission at $\gtrsim 6 \mu \mathrm{m}$ is still dominated by the AGN-powered hot-dust component. With the limited sample size, it is not clear whether the red WISE color at longer wavelengths is a common feature of hard X-ray-selected low-mass AGNs. However, we note that low-mass AGNs selected using other methods, such as the BPT diagram or the presence of the He II $\lambda 4686$ emission line, do not show as high a fraction of red $W 2-W 3$ colors (e.g., Figures 3 and 4 of Sartori et al. 2015; Figure 1 of Hainline et al. 2016) as our sample does, which might simply be due to the higher redshift and luminosities of our sample.

\section{2. $M_{\bullet}-M_{\star}$ and Eddington Ratios}

In our sample, five of the seven galaxies shown in Figure 4 exhibit broad $\mathrm{H} \alpha$ emission, allowing estimates of their black hole masses. The black hole mass for each broad-line AGN was estimated using Equation (5) of Reines et al. (2013) using the $\mathrm{H} \alpha$ line width and luminosity (Greene \& Ho 2005), with the updated $\mathrm{BH}$ radius-luminosity relation from Bentz et al. 


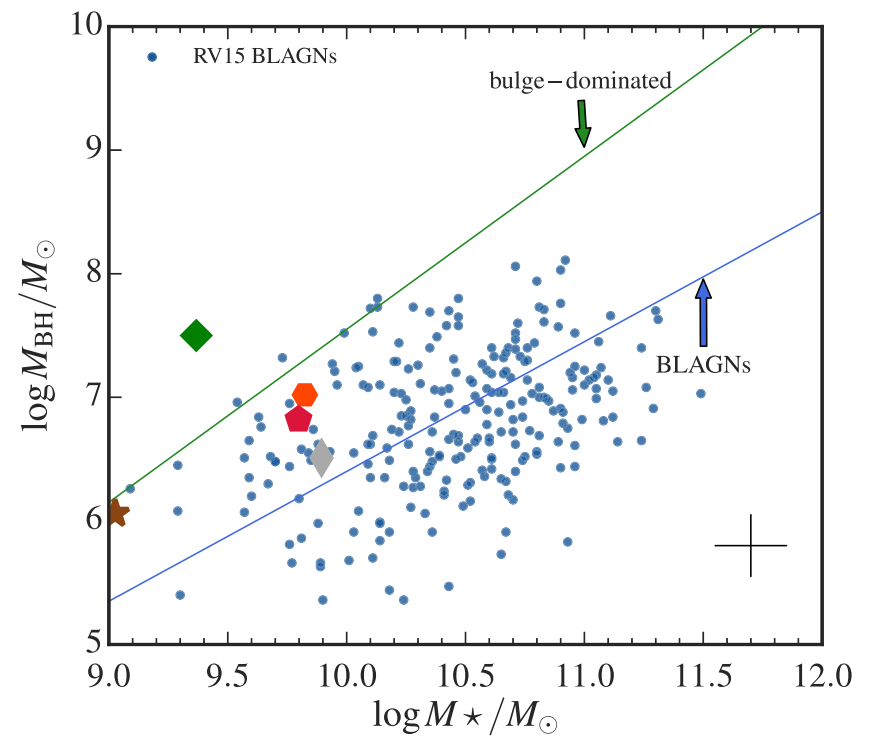

Figure 8. $M_{\bullet}-M_{\star}$ distribution for the five broad-line AGNs in our sample. For comparison, the $M_{\bullet}-M_{\star}$ distribution of the broad-line AGNs presented by Reines \& Volonteri (2015, RV15) is also shown. The best-fit linear relations for the RV15 AGN sample and the bulge-dominated galaxies from Kormendy \& Ho (2013, KH13) are also shown. Other large symbols are as in Figure 1. The typical $\sim 0.5$ dex systematic uncertainties of virial $\mathrm{BH}$ mass estimation (see, e.g., Reines \& Volonteri 2015) and the typical $\sim 0.3$ dex uncertainties of the $M_{\star}$ estimation are shown in the bottom right corner.

(2013):

$$
\begin{aligned}
\log \left(\frac{M_{\bullet}}{M_{\odot}}\right)= & \log \epsilon+6.57+0.47 \log \left(\frac{L_{\mathrm{H} \alpha}}{10^{42} \mathrm{erg} \mathrm{s}^{-1}}\right) \\
& +2.06 \log \left(\frac{\mathrm{FWHM}_{H_{\alpha}}}{10^{3} \mathrm{~km} \mathrm{~s}^{-1}}\right) .
\end{aligned}
$$

Here we choose the constant $\epsilon=1.075$, which is based on the mean virial factor of $\langle f\rangle=4.3$ (Onken et al. 2004; Grier et al. 2013). We show the $M_{\bullet}-M_{\star}$ distribution for the $N u S T A R$ low-mass galaxies in Figure 8. For comparison, we also show the best-fit linear relations from Reines \& Volonteri (2015) for 262 broad-line AGNs in the local universe $(z<0.055)$ and for the bulge-dominated galaxies with dynamical $M$. measurements from Kormendy \& Ho (2013). ${ }^{31}$

Overall, the $\log \left(M_{\bullet} / M_{\odot}\right)$ of the five broad-line AGNs in our NUSTAR-selected sample ranges from 6.1 to 7.5 , and thus all are more massive than the Reines \& Volonteri (2015) relation for broad-line AGNs. We also calculate the Eddington ratio for the five broad-line AGNs in our sample using intrinsic $L_{2-10 \mathrm{keV}}$ and a constant bolometric correction factor of 22.4 (Vasudevan \& Fabian 2007). We find that the Eddington ratio for these AGNs ranges from 3\% to 25\%. The high values of $M$. and Eddington ratio for the five broad-line objects in our sample are likely due to a combination of both the flux limits of $N U S T A R$ and the host-galaxy dilution effect, as less massive mBHs would either be missed in the current NuSTAR serendipitous survey or have their broad-line regions buried in the stellar continuum of the host galaxies (Hopkins et al. 2009). The Eddington ratios are also listed in Table 3.

\footnotetext{
31 We adopt the linear $M_{0}-M_{\star}$ relation for the bulge-dominated galaxies from Kormendy \& Ho (2013) calculated by Reines \& Volonteri (2015) (i.e., their Equation (6)).
}

\subsection{X-Ray Properties}

Here we compare the X-ray properties of our sample with those of existing low-mass AGNs reported in the literature. We emphasize that due to the challenges of detecting faint X-ray emission from mBHs, existing low-mass AGNs with X-ray observations are limited to a small number of low-redshift $(z \lesssim 0.2)$ objects, and an even smaller number of higherredshift objects $(z \lesssim 1$; e.g., Schramm et al. 2013; Pardo et al. 2016) are found in deep-survey regions. Since our sample is limited to $z \lesssim 0.3$, we assess our results by comparing the properties of our sample with two samples of low-mass AGNs in the local universe: the low-mass AGNs detected by Swift/ BAT, and those selected using broad optical emission lines.

We select the low-mass AGNs from the Swift/BAT AGNs studied in Koss et al. (2011, hereafter K11), which is a sample of 185 AGNs selected from the 22-month and 58-month Swift/ BAT all-sky surveys. The sensitivity limit of the K11 sample is $(1.1-1.5) \times 10^{-11} \mathrm{erg} \mathrm{cm}^{-2} \mathrm{~s}^{-1}$ in the $14-195 \mathrm{keV}$ band, which is equivalent to $(0.6-0.8) \times 10^{-11} \mathrm{erg} \mathrm{cm}^{-2} \mathrm{~s}^{-1}$ in NuSTAR-FB, assuming a typical AGN photon index of $\Gamma=1.8$. K11 also estimated the stellar mass for each AGN in their sample, which makes it possible for us to select AGNs with $M_{\star}$ similar to our low-mass NuSTAR sample. There are a total of 32 galaxies from K11 that could be considered as "lowmass" $\left(M_{\star} \lesssim 10^{10} M_{\odot}\right)$, similar to our NuSTAR sample. We note that the approach used to estimate the host-galaxy photometry and stellar mass is different in K11 compared to our approach outlined in Section 3. However, for simplicity, we directly adopt the values provided by K11 since we find that the $M_{\star}$ values derived using their approach and ours have little systematic difference (see Appendix B for a comparison between the quantities calculated using our approach and those directly obtained from K11).

For AGNs selected from low-mass galaxies based on the presence of AGN-powered optical emission lines, there have been a number of studies focusing on objects selected from the SDSS (e.g., Greene \& Ho 2007b; Barth et al. 2008; Dong et al. 2012b; Reines et al. 2013; Moran et al. 2014; Sartori et al. 2015), but only a fraction of these optical AGNs have soft X-ray follow-up observations. We focus on the two largest optical low-mass AGN samples with X-ray follow-up observations: the 50 AGNs with Chandra 2 ks snapshot observations discussed in Dong et al. (2012a) and the 14 AGNs with deeper XMM-Newton observations ( $>10 \mathrm{ks}$ ) from Ludlam et al. (2015). Both samples are selected from the 229 low-mass AGNs identified using SDSS DR4 (Greene \& Ho 2007b).

In Figure 9(a), we compare the $L_{10-40 \mathrm{keV}}$ values of our NUSTAR sample with those of the 32 low-mass galaxies selected from K11. The median $\log \left(L_{10-40 \mathrm{keV}} / \mathrm{erg} \mathrm{s}^{-1}\right)$ is $42.45 \pm 0.31$ for our NuSTAR sample and $42.88 \pm 0.15$ for the K11 subsample. The uncertainties for these median values were estimated using a bootstrapping analysis in which we randomly draw the samples with replacement 100 times. We also show the $L_{2-10 \mathrm{keV}}$ distributions of our NuSTAR sample and the optical low-mass AGN samples in Figure 9(b). The median $\log \left(L_{2-10 \mathrm{keV}} / \mathrm{erg} \mathrm{s}^{-1}\right)$ is $42.48 \pm 0.19$ for the NuSTAR sample and $42.10 \pm 0.09$ for the two optical samples.

We note that the redshift of the K11 sample is limited to $z<0.05$. However, beyond $z=0.05$, it is not likely for AGNs powered by accretion onto $\mathrm{mBHs}$ to emit hard X-ray emission exceeding the Swift/BAT sensitivity limit of $\approx 10^{-11}$ 

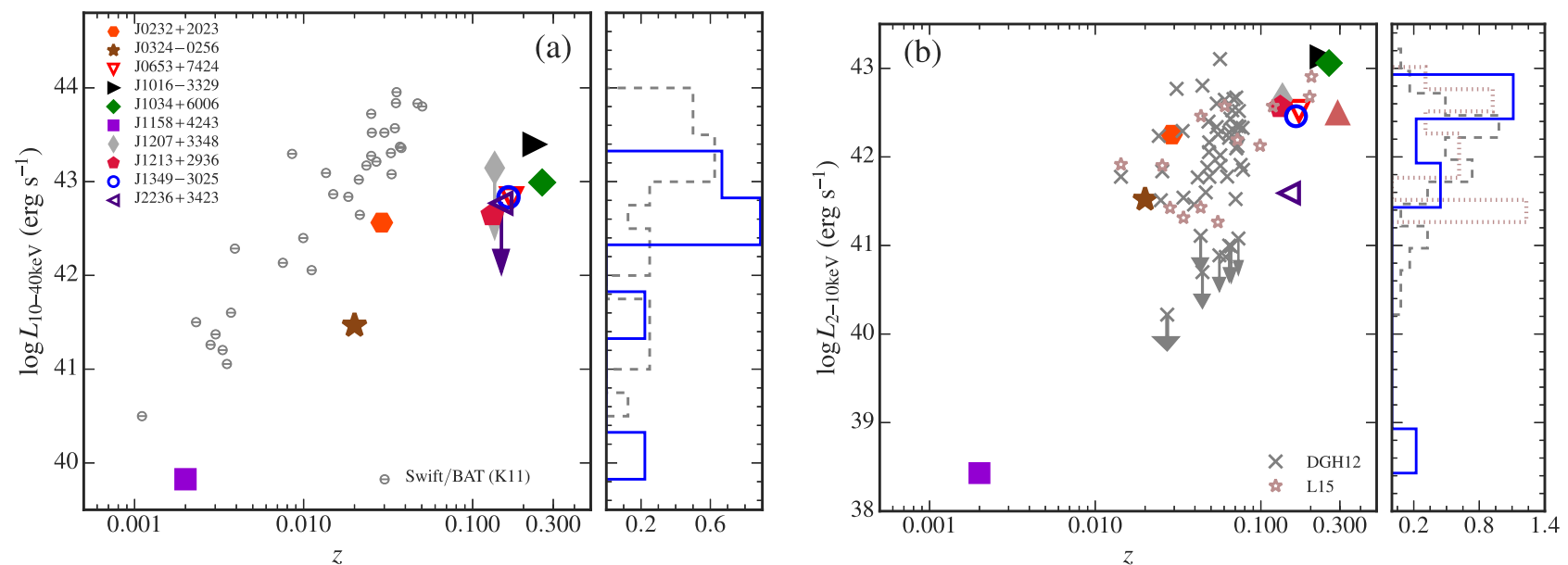

Figure 9. (a) Hard X-ray luminosities of the NuSTAR sample and the Swift/BAT sample from K11 (without correction for absorption). In the side panel, the normalized histogram of the NuSTAR sample is shown as the blue solid line, and the Swift/BAT sample is shown as the gray dashed line. (b) Rest-frame 2-10 keV X-ray luminosity (not corrected for absorption) distributions of the NuSTAR sample and the optical low-mass AGN samples from Dong et al. (2012a, DGH12) and Ludlam et al. (2015, L15). In the side panel, the normalized histogram of the NuSTAR sample is shown as the solid blue line, and the DGH12 and L15 samples are shown as the dashed and dotted lines, respectively.

ergs $\mathrm{cm}^{-2} \mathrm{~s}^{-1}$. For the NuSTAR-selected sample in this work, the average redshift is 0.14 , and the $L_{10-40 \mathrm{keV}}$ of the $N u S T A R$ sample is $\approx 0.4 \mathrm{dex}$ fainter than for the low-mass galaxies found in the K11 sample. This again highlights the excellent sensitivity of NUSTAR and its ability for studying low-mass galaxies beyond the local universe.

For the optically selected AGNs, Dong et al. (2012a) also targeted low-mass AGNs at low redshift $(z \lesssim 0.08)$, due to the flux limit of their $2 \mathrm{ks}$ Chandra snapshot observations. For the Ludlam et al. (2015) sample, the deeper XMM-Newton observations reach $z \lesssim 0.2$, which is more comparable to our NuSTAR sample. In Figure 9(b), we find that our NuSTAR sample occupies a similar region of $L_{2-10 \mathrm{keV}}-z$ parameter space to the X-ray follow-up observations of optical AGNs with broad emission lines and $M_{\bullet} \approx 10^{6} M_{\odot}$, demonstrating the strength of serendipitous $N U S T A R$ observations in detecting hard X-ray emission from low-mass AGNs in the low-redshift universe.

Some of the NUSTAR low-mass AGNs reported in this work are X-ray obscured (see Section 3.3). We note that there is not yet a clear understanding of the obscured AGN population hosted by low-mass galaxies, which is primarily due to the existing X-ray observations mostly having targeted AGNs with broad emission lines, and there are few X-ray-selected AGNs hosted by low-mass galaxies that are obscured to the best of our knowledge. With the NUSTAR-selected sample and the lowmass AGNs from K11, we can take a first step in constraining the X-ray-obscured fraction using hard X-ray-selected lowmass AGNs, although caution is required because the NuSTAR serendipitous survey is relatively shallow (with a median exposure time of $28 \mathrm{ks}$; see L17) and might still not be able to detect efficiently low-mass Compton-thick AGNs. We utilize the X-ray spectral analysis results for the NuSTAR sources and the ancillary soft X-ray data for the K11 AGNs from the literature (see Appendix C and Table 6). For X-ray-detected AGNs, $N_{\mathrm{H}}=10^{22} \mathrm{~cm}^{-2}$ is a commonly used value for separating X-ray type 1 and type 2 objects (but see also Merloni et al. 2014, for the use of slightly lower $N_{\mathrm{H}}$ values for classifying type 2 objects). We adopt this criterion to select X-ray type 2 AGNs from the NUSTAR and Swift/BAT samples. We find that the X-ray-obscured fraction for the seven objects in the NuSTAR sample with $N_{\mathrm{H}}$ measurements is $43_{-18}^{+15} \% .^{32}$ This obscuration is likely a lower limit as some of the most heavily obscured AGNs would not have been detected even with NuSTAR (e.g., Lansbury et al. 2014; Stern et al. 2014). For the K11 low-mass AGNs, the obscured fraction is $51 \% \pm 8 \%$. For the combined sample of 42 AGNs, we compute the obscured fraction to be $47_{-7}^{+8} \%$, but we caution that the two samples have different selection functions. The $N_{\mathrm{H}}$ versus $M_{\star}$ distributions for our sample and the K11 low-mass AGNs are shown in Figure 10.

While the accuracy of the obscured fraction presented here is limited by the small sample sizes and selection functions of the NUSTAR and K11 low-mass AGN samples, the results from the two samples are both broadly consistent with the findings of previous studies of the luminosity-dependent AGN obscured fraction. For example, the obscured fraction derived using Equation (5) from Lusso et al. (2013) is $\sim 50 \%$ for the median luminosity of our AGN sample. When we focus only on the nine dwarf galaxies with $M_{\star}<3 \times 10^{9} M_{\odot}$, there are only two X-ray-obscured AGNs (i.e., $N_{\mathrm{H}}>10^{22} \mathrm{~cm}^{-2}$ ) from the combined NuSTAR and K11 sample, which is equivalent to $22.2_{-8.1}^{+18.3 \%}$. To investigate this possible reduction of the obscured fraction, we separate the combined hard X-ray AGN sample into two subsamples, the "low-mass galaxies" with $3 \times 10^{9} M_{\odot}<M_{\star}<10^{10} M_{\odot}$, and the "dwarf galaxies" with $M_{\star}<3 \times 10^{9} M_{\odot}$. We apply a Peto-Prentice test on the $N_{\mathrm{H}}$ distributions for both subsamples to account for the upper limits on the $N_{\mathrm{H}}$ values (e.g., see Astronomy SURVival Analysis, Feigelson \& Nelson 1985). The two-sample PetoPrentice probability for both samples to follow the same distribution is $17.0 \%$.

\section{Discussion and Conclusions}

In this work, we present the first NuSTAR-selected sample of 10 low-mass galaxies harboring hard X-ray-emitting AGNs from the 40-month NUSTAR serendipitous survey (L17). Compared to low-mass AGNs in the previous-generation hard

\footnotetext{
32 We use the Cameron (2013) method to calculate the $68.3 \%$ binomial confidence limits of the obscured fraction.
} 
Table 6

Key Properties of Swift/BAT Low-mass Galaxies Selected from Koss et al. (2011)

\begin{tabular}{|c|c|c|c|c|c|c|c|}
\hline $\begin{array}{l}\text { Name } \\
\text { (1) }\end{array}$ & $\begin{array}{c}\text { R.A. } \\
(\mathrm{J} 2000) \\
(2)\end{array}$ & $\begin{array}{c}\text { Decl. } \\
(\mathrm{J} 2000) \\
(3)\end{array}$ & (4) & $\begin{array}{c}\log M_{\star} \\
\left(\log M_{\odot}\right) \\
\quad(5)\end{array}$ & $\begin{array}{l}\text { Swift/BAT ID } \\
\text { (6) }\end{array}$ & $\begin{array}{c}\log L_{10-40 \mathrm{keV}} \\
\left(\log \operatorname{erg~s}^{-1}\right) \\
(7)\end{array}$ & $\begin{array}{c}\log N_{\mathrm{H}} \\
\left(\log \mathrm{cm}^{-2}\right) \\
(8)\end{array}$ \\
\hline Mrk 352 & 14.972 & 31.8269 & 0.0149 & 9.65 & SWIFT J0059.4+3150 & 42.87 & 20.0 \\
\hline 2MASX J03534246+3714077 & 58.427 & 37.235 & 0.0183 & 9.9 & SWIFT J0353.7+3711 & 42.84 & 22.34 \\
\hline 2MASX J05054575-2351139 & 76.4405 & -23.8539 & 0.035 & 9.13 & SWIFT J0505.8-2351 & 43.84 & 22.85 \\
\hline MCG -05-14-012 & 85.8873 & -27.6514 & 0.0099 & 9.66 & SWIFT J0543.9-2749 & 42.4 & 20.0 \\
\hline 2MASX J06411806+3249313 & 100.3252 & 32.8254 & 0.047 & 9.94 & SWIFT J0641.3+3257 & 43.83 & 23.3 \\
\hline Mrk 1210 & 121.0244 & 5.1138 & 0.0135 & 9.89 & SWIFT J0804.2+0507 & 43.09 & 23.0 \\
\hline Mrk 18 & 135.493 & 60.152 & 0.0111 & 9.57 & SWIFT J0902.0+6007 & 42.06 & 23.3 \\
\hline 2MASX J09043699+5536025 & 136.154 & 55.6008 & 0.037 & 9.76 & SWIFT J0904.3+5538 & 43.37 & 20.78 \\
\hline 2MASX J09112999+4528060 & 137.8749 & 45.4683 & 0.0268 & 9.76 & SWIFT J0911.2+4533 & 43.21 & 23.48 \\
\hline IC 2461 & 139.992 & 37.191 & 0.0075 & 9.54 & SWIFT J0920.1+3712 & 42.13 & 22.85 \\
\hline Mrk 110 & 141.3036 & 52.2863 & 0.0353 & 9.9 & SWIFT J0925.0+5218 & 43.95 & 20.3 \\
\hline CGCG 122-055 & 145.52 & 23.6853 & 0.0214 & 9.94 & SWIFT J0942.2+2344 & 42.65 & 20.1 \\
\hline NGC 3079 & 150.4908 & 55.6798 & 0.0037 & 9.98 & SWIFT J1001.7+5543 & 41.6 & 22.3 \\
\hline NGC 3227 & 155.8774 & 19.8651 & 0.0039 & 9.98 & SWIFT J1023.5+1952 & 42.29 & 22.3 \\
\hline ARP 151 & 171.4007 & 54.3825 & 0.0211 & 9.71 & SWIFT J1125.6+5423 & 43.02 & 21.7 \\
\hline NGC 3718 & 173.1452 & 53.0679 & 0.0033 & 9.98 & SWIFT J1132.7+5301 & 41.2 & 22.0 \\
\hline $\mathrm{MCG}+10-17-061$ & 176.3881 & 58.9781 & 0.0099 & 9.8 & SWIFT 1145.2+5905 & 42.55 & 22.9 \\
\hline NGC 4051 & 180.7901 & 44.5313 & 0.0023 & 9.44 & SWIFT J1203.0+4433 & 41.5 & 20.0 \\
\hline NGC 4102 & 181.5963 & 52.7109 & 0.0028 & 9.68 & SWIFT J1206.2+5243 & 41.26 & 24.48 \\
\hline NGC 4138 & 182.3741 & 43.6853 & 0.003 & 9.61 & SWIFT J1209.4+4340 & 41.37 & 23.0 \\
\hline Mrk 50 & 185.8506 & 2.6791 & 0.0234 & 9.9 & SWIFT J1223.7+0238 & 43.17 & 20.0 \\
\hline NGC 4395 & 186.4538 & 33.5468 & 0.0011 & 8.28 & SWIFT J1202.5+3332 & 40.5 & 21.3 \\
\hline ESO 506-G027 & 189.7275 & -27.3078 & 0.025 & 9.84 & SWIFT J1238.9-2720 & 43.72 & 23.9 \\
\hline SBS $1301+540$ & 195.9978 & 53.7917 & 0.0299 & 9.79 & SWIFT J1303.8+5345 & 43.52 & 22.3 \\
\hline NGC 5273 & 205.5347 & 35.6542 & 0.0035 & 9.64 & SWIFT J1341.9+3537 & 41.06 & 20.0 \\
\hline UM 614 & 207.4701 & 2.0791 & 0.0327 & 9.99 & SWIFT J1349.7+0209 & 43.3 & 21.0 \\
\hline Mrk 464 & 208.973 & 38.5746 & 0.0501 & 9.67 & SWIFT J1356.1+3832 & 43.8 & 24.0 \\
\hline Mrk 477 & 220.1587 & 53.5044 & 0.0377 & 9.87 & SWIFT J1441.4+5341 & 43.36 & 22.95 \\
\hline NGC 5995 & 237.104 & -13.7578 & 0.0252 & 9.89 & SWIFT J1548.5-1344 & 43.52 & 22.0 \\
\hline CGCG 300-062 & 265.8225 & 62.8392 & 0.033 & 9.9 & SWIFT J1743.4+6253 & 43.08 & 23.0 \\
\hline 2MASX J21355399+4728217 & 323.975 & 47.4727 & 0.025 & 9.41 & SWIFT J2156.1+4728 & 43.27 & 21.6 \\
\hline KAZ 320 & 344.8871 & 24.9182 & 0.0345 & 9.7 & SWIFT J2259.7+2458 & 43.57 & 20.0 \\
\hline
\end{tabular}

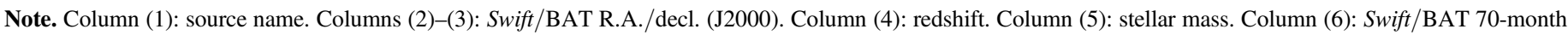

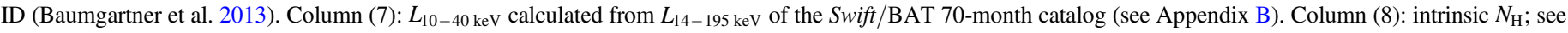
Appendix B for details.

X-ray Swift/BAT survey, our NuSTAR-selected sample includes several low-redshift objects with much lower hard X-ray luminosities, as well as low-mass AGNs at higher redshifts. The soft X-ray luminosities of our objects are consistent with those of optically selected low-mass AGNs in the low-redshift universe. We find that $30_{-10}^{+17} \%$ of the NuSTAR AGNs in our sample do not have AGN-powered highionization lines in their optical spectra, which demonstrates the capability of NUSTAR for detecting a low-mass AGN population that might be missed in wide-area optical surveys.

We also study the WISE colors of our sample and find that the majority of the X-ray-obscured AGNs and optically normal AGNs in our sample do not have red $W 1-W 2$ colors similar to those of luminous mid-IR AGNs. We note that eight of the 10 low-mass NUSTAR galaxies still show red mid-IR colors at longer mid-IR wavelengths $(W 2-W 3>3)$ that are likely due to the presence of hot dust powered by AGNs. However, a substantial number of low-mass star-forming galaxies also exhibit red WISE W2-W3 color (Hainline et al. 2016), suggesting that the effectiveness of using only the red WISE colors to select low-mass AGNs missed by optical surveys is limited.

We constrain the obscured fraction of hard X-ray-selected low-mass AGNs by combining our sample with the K11 Swift/
BAT low-mass AGNs. We find that hard X-ray-selected lowmass AGNs have an "obscured fraction" of $47_{-7}^{+8} \%$. This is consistent with the obscured fraction extrapolated from studies of the luminosity-dependent fraction of obscured AGNs (e.g., Equation (5) of Lusso et al. 2013). However, when focusing on the "dwarf galaxies" $\left(M_{\star}<3 \times 10^{9} M_{\odot}\right)$, the fraction of AGNs with $N_{\mathrm{H}}>10^{22} \mathrm{~cm}^{-2}$ drops to $22.2_{-8.1}^{+18.3} \%$ (or $2 / 9$ ).

Notably, the two heavily obscured AGNs hosted by dwarf galaxies (J115851+4243.2 from our sample and J0505.8-2351 from K11) do not have blue host-galaxy colors similar to the high-redshift galaxies with stacked X-ray spectra that suggest the presence of heavily obscured AGNs (Xue et al. 2012; Mezcua et al. 2016). The likely reason is that the obscured AGN population suggested by the high-redshift X-ray stacking studies of star-forming galaxies is still less luminous (e.g., $\left\langle L_{2-10 \mathrm{kev}}\right\rangle \approx 4.8 \times 10^{40} \mathrm{erg} \mathrm{s}^{-1}$ for "sample D" in Xue et al. 2012 , and $L_{2-10 \mathrm{keV}} \lesssim 6 \times 10^{40} \mathrm{erg} \mathrm{s}^{-1}$ for the Mezcua et al. 2016 sample) than the majority of our sample and thus is not detected in the NUSTAR serendipitous survey.

On the other hand, several recent studies have found that AGNs in dwarf galaxies selected based on optical emission line ratios have a high Seyfert 2 fraction (e.g., Reines et al. 2013; 


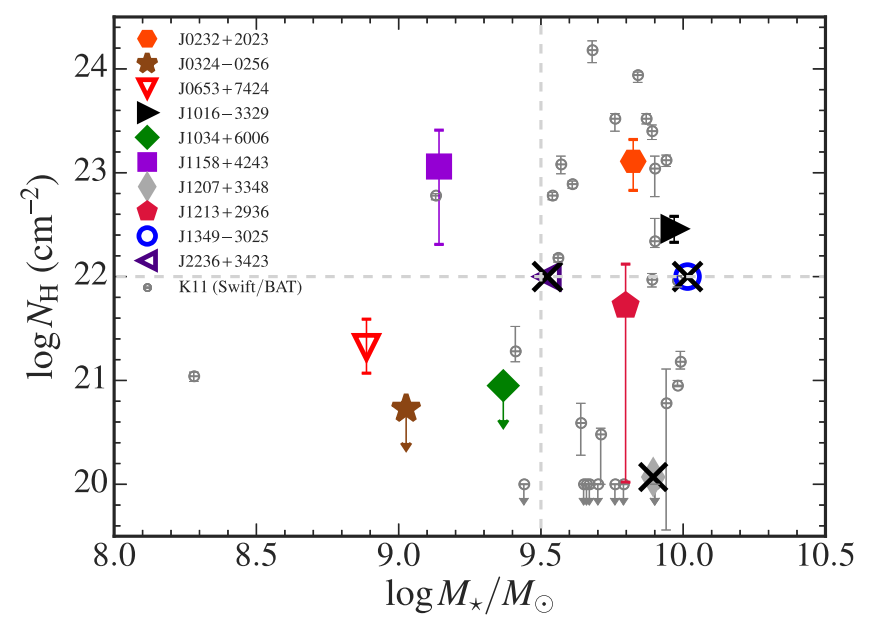

Figure 10. $N_{\mathrm{H}}$ vs. $M_{\star}$ for low-mass AGNs selected using NuSTAR and Swift/ BAT (gray dots). The $N_{\mathrm{H}}$ values derived based on X-ray spectral fitting are shown as the symbols with vertical error bars that correspond to a $90 \%$ confidence interval. Sources with $N_{\mathrm{H}}$ values estimated based on their hardness ratio are shown as crosses without vertical error bars. See Section 3.3 for details. The typical uncertainty in $M_{\star}$ is 0.3 dex (see Section 3.1). We note that there are only two $N_{\mathrm{H}}>10^{22} \mathrm{~cm}^{-2}$ AGNs hosted by dwarf galaxies with $\log M_{\star} / M_{\odot}<9.5$, which highlights the limited sample size of X-ray-obscured AGNs in dwarf galaxies.

Moran et al. 2014). Some studies have also suggested that lowluminosity Seyfert 2 galaxies are unobscured and lack broadline regions as a result of falling below a critical accretion luminosity that is independent of the Eddington rate of the accreting mBH (e.g., Bianchi et al. 2008; Trump et al. 2011; Elitzur et al. 2014; Elitzur \& Netzer 2016). Therefore, X-ray observations are still essential to determine whether a lowluminosity AGN is obscured by intervening gas/dust. For AGNs that are more heavily obscured, hard X-ray observations provide arguably the best constraint on whether a lowluminosity AGN is obscured. Although the current sample size of hard X-ray-selected AGNs hosted by dwarf galaxies is still limited, our results have demonstrated the capability of NUSTAR in detecting heavily obscured AGNs hosted by dwarf galaxies. We note that the current serendipitous-survey catalog (L17) from which our low-mass AGN sample is drawn is based on the 40-month NUSTAR observations with $\approx 50 \%$ optical spectroscopic coverage. Therefore, a $10 \mathrm{yr}$ NuSTAR serendipitous survey with complete optical spectroscopic follow-up observations will likely increase the sample size of NuSTARdetected AGNs in low-mass galaxies by more than a factor of five compared to what is presented here. But even a $10 \mathrm{yr}$ serendipitous survey may have only a few heavily obscured AGNs (similar to our J115851+4243.2), motivating targeted follow-up NUSTAR observations of heavily obscured AGNs selected at other wavelengths to build a more complete picture of the AGN population in dwarf galaxies.

In conclusion, this small sample of NUSTAR-selected lowmass AGNs has demonstrated that NUSTAR is capable of detecting a variety of AGNs in low-mass galaxies that are complementary to the existing emission-line low-mass AGNs that are found in optical surveys and previous-generation hard $\mathrm{X}$-ray surveys. We stress that the spectroscopic observations and ancillary soft X-ray data are instrumental in the construction of our NuSTAR-selected sample. With the small volume of the current NUSTAR surveys, the most efficient method of systematically searching for low-mass AGNs that are not broad emission line AGNs may still be cross-matching soft X-ray observations with optical spectroscopic surveys of galaxies. However, we note that there are only three objects in our sample with SDSS spectra, which is due to the flux limits $(r<17.77$ for the main galaxy targets) for spectroscopic observations by the SDSS. Moreover, a recent study that matched the Chandra Source Catalog with local dwarf galaxies in the SDSS is limited to the local universe and has primarily found low-luminosity X-ray sources that are not likely to be only associated with AGN activity (Lemons et al. 2015). With a greatly improved survey volume, the upcoming eROSITA all-sky X-ray survey (Merloni et al. 2012) and next-generation wide-area spectroscopic surveys such as the Subaru PFS survey (Takada et al. 2012) should reveal many more X-ray AGNs hosted by dwarf galaxies at moderate redshifts, as these surveys will reach flux limits that are deep enough to recover the majority of sources similar to the NuSTAR objects reported in this work. However, as some of the targets in our sample are heavily obscured, NUSTAR remains a key observatory for providing insights about low-mass AGNs that are obscured by Compton-thick column densities similar to the megamaser AGNs.

We thank the referee for carefully reading the manuscript and providing helpful comments. This work was supported under NASA contract no. NNG08FD60C and made use of data from the $N U S T A R$ mission, a project led by the California Institute of Technology, managed by the Jet Propulsion Laboratory, and funded by the National Aeronautics and Space Administration. We thank the NuSTAR Operations, Software and Calibration teams for support with the execution and analysis of these observations. This research has made use of the NUSTAR Data Analysis Software (NuSTARDAS) jointly developed by the ASI Science Data Center (ASDC, Italy) and the California Institute of Technology (USA). C.-T.J.C. and W. N.B. acknowledge support from Caltech $N u S T A R$ subcontract 44A-1092750. Support for A.E.R. was provided by NASA through Hubble Fellowship grant HST-HF2-51347.001-A awarded by the Space Telescope Science Institute, which is operated by the Association of Universities for Research in Astronomy, Inc., for NASA, under contract NAS 5-26555. D. M.A. gratefully acknowledges support from Science and Technology Facilities Council (ST/L00075X/1). F.E.B. and C.R. acknowledge support from NASA NuSTAR A01 Award NNX15AV27G, CONICYT-Chile grants Basal-CATA PFB06/2007, FONDECYT Regular 1141218 and 1151408, ChinaCONICYT Fellowship, and the Ministry of Economy, Development, and Tourisms Millennium Science Initiative through grant IC120009, awarded to the Millennium Institute of Astrophysics, MAS. This publication makes use of data products from the Two Micron All Sky Survey, which is a joint project of the University of Massachusetts and the Infrared Processing and Analysis Center/California Institute of Technology, funded by the National Aeronautics and Space Administration and the National Science Foundation. Funding for SDSS-III has been provided by the Alfred P. Sloan Foundation, the Participating Institutions, the National Science Foundation, and the U.S. Department of Energy Office of Science. The SDSS-III Web site is http://www.sdss3.org/. This research has made use of the "Aladin sky atlas" developed at CDS, Strasbourg Observatory, France (Bonnarel et al. 2000). This work has also made use of observations made with the Spitzer Space Telescope, obtained from the NASA/IPAC 


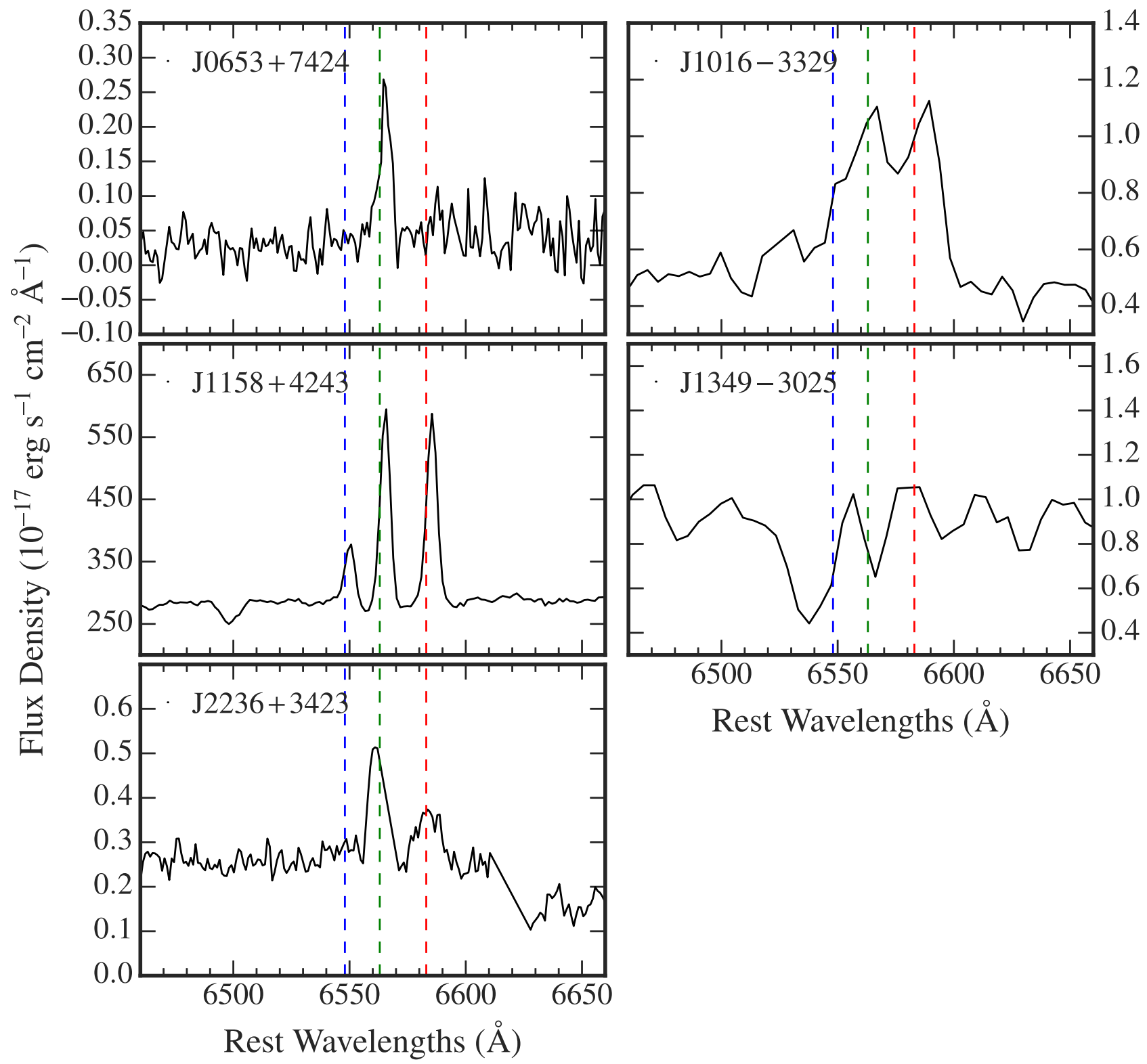

Figure 11. Spectra for objects without broad $\mathrm{H} \alpha$ lines plotted in the same rest-frame wavelength range as Figure 3 . In each panel, the emission lines [N II] $\lambda 6548, \mathrm{H} \alpha$ $\lambda 6563$, and [N II] $\lambda 6583$ are marked as the blue, green, and red dashed lines, respectively. We note that J101609-3329.6 and J115851+4243.2 still have strong narrow $\mathrm{H} \alpha$ lines, but the best-fit broad $\mathrm{H} \alpha$ component for these two objects does not exceed the $500 \mathrm{~km} \mathrm{~s}^{-1}$ threshold we used to identify broad-line AGNs. For J065318 +7424.8 and J134934-3025.5, the rest-frame wavelength for $\mathrm{H} \alpha$ emission lines coincides with the telluric A-band lines.

Infrared Science Archive, both of which are operated by the Jet Propulsion Laboratory, California Institute of Technology, under a contract with the National Aeronautics and Space Administration.

Facilities: NuSTAR, Chandra, XMM-Newton, Swift/BAT, Swift/XRT, WISE, Keck, NTT, Palomar.

Software: Astropy, CIAO, HEAsoft, XMMSAS, XSPEC.

\section{Appendix A \\ Notes on Individual Galaxies}

Here we briefly summarize the multiwavelength properties and describe the procedures of X-ray spectral analysis for each object in our sample. The resulting X-ray and optical spectra and other relevant information for each object are shown in the online figure set (Figure 2).
$J 023229+2023.7$. - This galaxy has the optical spectrum of a typical broad-line AGN. However, the NUSTAR spectrum suggests the presence of a substantial amount of absorption in the X-rays. For both available XMM-Newton observations, the position of J023229+2023.7 is located near the XMM-Newton chip gap of the PN detector and on the dead CCD of the MOS1 detectors, and they are both severely affected by background flaring. Data from the MOS2 detector were taken under the timing mode, and there is no useful spectral information. We only consider the NUSTAR data for X-ray spectral analysis. We first fit the data with an unabsorbed power law. The resulting power-law photon index of $\Gamma=1.2$ and the residuals at $\approx 6.4 \mathrm{keV}$ suggest the presence of absorption and an $\mathrm{Fe} \mathrm{K} \alpha$ line component. Thus, we fit the spectrum again with an absorbed power-law model with an $\mathrm{Fe} \mathrm{K} \alpha$ line at rest frame $6.4 \mathrm{keV}$. The intrinsic photon index is fixed at 1.8 to stop convergence at low values due to the degeneracy with $N_{\mathrm{H}}$. The 
resulting column density is $1.4_{-0.6}^{+0.8} \times 10^{23} \mathrm{~cm}^{-2}$, and the $\mathrm{Fe}$ $\mathrm{K} \alpha$ line equivalent width is $\approx 0.5 \mathrm{keV}$. We note that the significant equivalent width of the $\mathrm{Fe} \mathrm{K} \alpha$ line and the residuals above $>20 \mathrm{keV}$ may imply that $\mathrm{J} 023229+2023.7$ has an additional Compton-reflection component.

J032459-0256.2. - This object has already been identified as a dwarf galaxy in Alexander et al. (2013) with $M_{\star}=2 \times 10^{9} M_{\odot}$. Its soft X-ray luminosity, $L_{2-10 \mathrm{keV}}=3.3 \times 10^{41} \mathrm{erg} \mathrm{s}^{-1}$, is derived by jointly fitting the available NuSTAR and Swift/XRT data. We find that the X-ray spectrum of J032459-0256.2 is consistent with an absorbed power law with $\Gamma=1.9_{-0.2}^{+0.3}$ and intrinsic $N_{\mathrm{H}}<3.4 \times 10^{21} \mathrm{~cm}^{-2}$. Although its $2-10 \mathrm{keV} \mathrm{X-ray} \mathrm{lumin-}$ osity does not exceed the $10^{42}$ erg s $\mathrm{s}^{-1}$ threshold, its optical spectrum exhibits strong broad $\mathrm{H} \alpha$ and narrow high-ionization emission lines powered by an AGN. The BH mass of this object is $\log M_{\bullet} / M_{\odot}=6.06$ based on its broad $\mathrm{H}_{\alpha}$ emission. With the clear optical AGN signatures, we consider J032459-0256.2 to be a bona fide dwarf AGN.

J065318+7424.8. - NuSTAR J065318+7424.8 is a faint emission line galaxy with $\log M_{\star} / M_{\odot}=8.9$. Since this object has sufficient photons from both NuSTAR and Chandra, we jointly fit the NuSTAR and Chandra data. We find that its X-ray spectrum is consistent with an absorbed power law with modest intrinsic column density $\left(N_{\mathrm{H}}=2.1_{-1.2}^{+1.8} \times 10^{21} \mathrm{~cm}^{-2}\right)$, suggesting that the lack of AGN-powered emission lines is not due to the presence of heavy obscuration. The soft X-ray luminosity for this object is $L_{2-10 \mathrm{keV}} \approx 3.0 \times 10^{42} \mathrm{erg} \mathrm{s}^{-1}$, suggesting that $\mathrm{J} 065318+7424.8$ is indeed powered by accretion onto a massive $\mathrm{BH}$.

J101609-3329.6.-Based on its optical spectrum, J101609-3329.6 is a narrow-line Seyfert galaxy (type 2). To explore whether its X-ray properties are consistent with those of a type 2 AGN, we first examine the archival Chandra data of J101609-3329.6. The $10 \mathrm{ks}$ Chandra image reveals that there are two point sources with $\approx 5^{\prime \prime}$ separation, and only one of the point sources has significant $>2 \mathrm{keV} \mathrm{X}$-ray photons. Thus, we consider the Chandra point source with more hard X-ray photons to be the correct counterpart of the AGN in J101609-3329.6. Although the Chandra photon counts are too low for spectral analysis, the Chandra $2-8 \mathrm{keV}$ to $0.5-2 \mathrm{keV}$ photon count ratio suggests that the AGN in J101609-3329.6 is obscured. We next extracted the XMMNewton pn spectrum for J101609-3329.6 with a 10" radius and jointly fit the NuSTAR data with the XMM-Newton data. The 2-24 keV spectrum could be fitted with an absorbed power law with $\Gamma=1.28 \pm 0.3$ and $N_{\mathrm{H}} \approx 2.9 \pm 1.0 \times 10^{22} \mathrm{~cm}^{-2}$, supporting the indication from the Chandra band ratio and the lack of broad emission lines in the optical spectrum.

$J 103410+6006.7 .-\mathrm{J} 103410+6006.7$ is also an optical broad-line AGN. The NuSTAR photon counts of J103410 +6006.7 are not sufficient for spectral fitting. Therefore, we only consider the XMM-Newton data for this object. The XMMNewton spectrum is consistent with a typical unabsorbed AGN with $\Gamma=1.7 \pm 0.2$ and $N_{\mathrm{H}}<8.9 \times 10^{20} \mathrm{~cm}^{-1}$ (90\% upper limit). Among the broad-line AGNs in our NuSTAR low-mass galaxy sample, J103410+6006.7 has the highest ratio of black holes to galaxy mass, which places J103410+6006.7 in the $M$. $-M_{\star}$ region occupied by the local bulge-dominated galaxies.

$J 115851+4243.2$. - J115851+4243.2, also known as IC 750, is the lowest-redshift galaxy in our sample $(z=0.002)$. The $L_{2-10 \mathrm{keV}}=2.7 \times 10^{38} \mathrm{erg} \mathrm{s}^{-1}$ is calculated from the publicly available on-axis Chandra observation. In detail, we first visually inspected the publicly available Chandra and XMM-Newton images and found that J115851 +4243.2 appears to be extended in both Chandra $(0.5-8 \mathrm{keV})$ and XMM-Newton (0.2-12 keV) images. Considering the higher-resolution Chandra image, we find that the $>2 \mathrm{keV}$ $\mathrm{X}$-ray emission primarily originates from a central $\approx 2^{\prime \prime}$ region that coincides with the SDSS fiber location $(<0$ !' 3 separation). There are also two additional off-nuclear X-ray sources $\approx 5^{\prime \prime}$ away from the optical position. In the lower-energy bands $(E<2 \mathrm{keV})$, the Chandra image appears more extended. Considering the XMM-Newton image, the central nucleus cannot be distinguished from the off-nuclear point sources seen by Chandra. This is likely the reason that the XMM-Newton 3-8 keV flux is higher than the Chandra 3-8 keV flux (see Table 4 ) by a factor of $\sim 1.8$. Thus, we extract the Chandra spectrum from the central $2^{\prime \prime}$ region to avoid possible contamination from off-nuclear X-ray binaries. The Chandra spectrum still requires an additional diffuse thermal plasma component (we use the XSPEC VMEKAL model in addition to an absorbed power law, i.e., the following XSPEC model: TBABS $\times($ TBABS $\times$ VMEKAL + TBABS $\times$ ZPOW $))$ to have an acceptable fit of the $0.5-8 \mathrm{keV}$ spectrum. With a fixed photon index $\Gamma=1.8$, we obtain a best-fit column density of $1.2_{-1.0}^{+1.4} \times 10^{23}$ $\mathrm{cm}^{-2}\left(\chi^{2}=58.27 / 71\right)$, suggesting that the AGN in J115851 +4243.2 is heavily obscured and has an intrinsic X-ray luminosity of $L_{2-10 \mathrm{keV}}^{\text {int }}=5.4 \times 10^{40} \mathrm{erg} \mathrm{s}^{-1}$.

With the low $L_{2-10 \mathrm{keV}}$ of $\mathrm{J} 115851+4243.2$, the best-fit lineof-sight column density is still insufficient to promote the intrinsic X-ray luminosity to the range of typical AGNs. However, as we have briefly discussed in Section 3.4, the optical emission line ratios of $\mathrm{J} 115851+4243.2$ strongly suggest the presence of an AGN. Even though the X-ray luminosity of $\mathrm{J} 115851+4243.2$ is low, it is not likely for a typical X-ray binary to produce ionizing photons that would push the emission-line ratios of $\mathrm{J} 115851+4243.2$ to the observed values (see Figure 4). Moreover, the angular proximity of the Chandra position and the SDSS position ( $\lesssim 0$ !! 3) shows that the physical separation between the X-ray point source and the optical centroid is less than $50 \mathrm{pc}$ at $z=0.002$, which further reduces the likelihood of $\mathrm{J} 115851$ +4243.2 being an off-nuclear ULX. The luminous nuclear mid-IR emission based on the WISE photometry and the old stellar population suggested by the optical spectrum of J115851 +4243.2 also support the presence of a heavily obscured AGN (see Section 4.3). Therefore, we argue that J115851+4243.2 is indeed powered by accretion onto the central massive $\mathrm{BH}$.

$J 120711+3348.5$ and $J 121358+2936.1$. - J120711+3348.5 and $\mathrm{J} 121358+2936.1$ are both broad-line AGNs with many similar properties, including redshifts, $W 1-W 2$ colors, and M. While the NUSTAR photon counts for both objects are limited, J121358+2936.1 has a higher flux in the NuSTAR hard band than J120711+3348.5. For J120711+3348.5, we examine the existing Swift/XRT data and find that it has only 10 photon counts above $>2 \mathrm{keV}$. Thus, we cannot reliably constrain its obscuring column density. For J121358+2936.1, we fit the publicly available Chandra data with an absorbed power law and found that the best-fit model has a moderate obscuring column density $\left(N_{\mathrm{H}} \lesssim 10^{22} \mathrm{~cm}^{-2}\right)$.

J134934-3025.5.-For J134934-3025.5, the XMM-Newton archival data have only 20 photon counts in the $2-10 \mathrm{keV}$ band of EPIC pn, which is not sufficient for spectral fitting. We 
estimate its $L_{2-10 \mathrm{keV}}$ to be $\approx 2.9 \times 10^{42} \mathrm{erg} \mathrm{s}^{-1}$ based on its $X M M$-Newton photon count rate, assuming an AGN photon index of 1.8 due to the limited photon counts. In this luminosity range, J134934-3025.5 is considered to be a bona fide X-ray AGN. However, its optical spectrum exhibits a significant $4000 \AA$ break and absorption lines in $\mathrm{Ca} \mathrm{H}, \mathrm{K}$, and $\mathrm{H} \alpha$. This strongly suggests that $\mathrm{J} 134934-3025.5$ is a quiescent galaxy. As we have discussed at length in Section 4.2, future X-ray follow-up observations are required to determine the reason for the lack of optical emission lines in J134934-3025.5.

$J 223654+3423.5 .-\mathrm{J} 223654+3423.5$ is a faint emission line galaxy. Notably, its soft X-ray luminosity $\left(L_{2-10 \mathrm{kev}}=\right.$ $3.8 \times 10^{41} \mathrm{erg} \mathrm{s}^{-1}$, estimated based on the Chandra observation with a fixed photon index of 1.8 using PIMMS) is slightly lower than the $10^{42} \mathrm{erg} \mathrm{s}^{-1}$ limit for empirically separating AGNs from ULXs. While it is still possible for J223654 +3423.5 to be an extremely luminous ULX, we note that ULXs with luminosities similar to that of J223654+3423.5 are likely to be powered by accretion onto off-nuclear mBHs (e.g., Walton et al. 2014; Mukherjee et al. 2015). Without highresolution spatial information on the source of X-ray emission, the distinction between the $\mathrm{mBH}$-powered ULX and low-mass AGN becomes ambiguous. However, this should not affect the primary objective of this work of searching for accreting $\mathrm{mBHs}$ using hard X-ray observations. Moreover, the NuSTAR softband luminosity is $\approx 6$ times higher than that for the Chandra observation for $\mathbf{J} 223654+3423.5$, suggesting that the $L_{2-10 \mathrm{keV}}$ is more luminous than $10^{42} \mathrm{erg} \mathrm{s}^{-1}$ during the time of the NuSTAR observation. Therefore, we consider J223654+3423.5 to be a low-mass AGN powered by an $\mathrm{mBH}$ similar to the other objects in our sample. We note that the separation between the NuSTAR and Chandra observation dates is relatively short (56 days), but the origin of the variability of $\mathrm{J} 223654+3423.5$ could not be determined with the currently available data.

\section{Appendix B \\ Notes on Additional Soft X-ray Observations Not Used in This Work}

In Section 3.3 and Table 4, we report that there are six sources in our sample with more than one soft X-ray observation. After careful considerations, these observations were not used in this work. Here we present the details of these observations.

J023229+2023.7.-The XMM-Newton PN data of obsIDs 0604210201 and 0604210201 both suffer from significant high-energy (10-12 keV) background flaring for more than $50 \%$ of the observed duration, and both observations have less than $10 \mathrm{ks}$ effective PN exposure time. Additionally, the source is located near the PN chip gap for both obsIDs. While the less sensitive MOS detectors were not as severely affected by the flaring background, J023229+2023.7 is located on the MOS1 CCD that has been permanently shut off. The data on MOS2 were taken under "timing mode" for both obsIDs, and we could not extract useful spectral information for J023229+2023.7. Therefore, we only adopt the $N u S T A R$ data for constraining the AGN X-ray property.

J032459-0256.2.-We extract the EPIC pn spectrum from the XMM-Newton observation obsID 0405240201 using the similar approach described in Section 3.3. We jointly fit the XMM-Newton data and the NUSTAR data following the description of this source given in Appendix A and find the best-fit parameters to be within the uncertainty range of the joint Swift/XRT and NuSTAR fit. The best-fit X-ray luminosity is $L_{2-10 \mathrm{keV}}=2.3 \times 10^{41} \mathrm{erg} \mathrm{s}^{-1}$, which is slightly less than the result based on the Swift/XRT and NuSTAR data $\left(L_{2-10 \mathrm{keV}}=3.3 \times 10^{41} \mathrm{erg} \mathrm{s}^{-1}\right)$. For this work, we choose to use the result based on the Swift/XRT and NuSTAR data because its $3-8 \mathrm{keV}$ X-ray flux is closer to that of the NuSTAR data.

J065318+7424.8.-The XMM-Newton observation obsID 0061540101 has only $\sim 7 \mathrm{ks}$ background-filtered exposure time; thus, no useful spectral information is available. The $X M M-N e w t o n$ data 0144230101 have $\sim 30 \mathrm{ks}$ exposure time, but the XMM-Newton image appears to be extended. We compare the spectrum of 0144230101 with the best-fit model based on the $\sim 70 \mathrm{ks}$ Chandra data and find that 0144230101 has significant excess below $3 \mathrm{keV}$, suggesting that the $X M M$ Newton spectrum might be contaminated by the extended soft $\mathrm{X}$-ray emission. To avoid large uncertainties due to the requirement of an additional soft X-ray component and the smaller photon counts of the XMM-Newton observation, we use only the Chandra data for this work and do not consider the additional data from 0144230101 .

J101609-3329.6.- The exposure time of the additional Chandra data for J101609-3329.6 is only $\sim 10 \mathrm{ks}$, and the photon counts are too low to provide useful spectral constraints compared to the $\sim 80 \mathrm{ks} X M M$-Newton observation adopted for the main analysis.

$J 115851+4243.2$. - As described in Appendix A, there are multiple Chandra point sources within the $\sim 10^{\prime \prime}$ XMM-Newton spectrum extraction region for the XMM-Newton data 074404301. To avoid contaminations from these off-nuclear sources, we do not make use of the XMM-Newton observation.

$J 223654+3423.5$.- This source has three different $10 \mathrm{ks}$ Chandra observations, and none of them have sufficient photon counts for spectral analysis. For the main article, we choose obsID 17570 because the flux of this obsID is the closest to that of the NuSTAR observation. Further investigating the nature of the X-ray variability of $\mathrm{J} 223654+3423.5$ would require additional X-ray observations.

\section{Appendix C \\ Notes on the Swift/BAT Low-mass Galaxies}

Here we briefly summarize the properties of the Swift/BAT low-mass AGNs discussed in this paper. K11 selected local $(z \lesssim 0.05)$ AGNs from the 22-month and 58-month Swift/BAT catalogs in the northern sky (Decl. $>-25 \mathrm{deg}$ ). For galaxies without SDSS photometry, K11 observed them using the Kitt Peak $4 \mathrm{~m}$ telescope with the same filters as those of SDSS. The nuclear contribution to the photometry was then removed for each galaxy using surface-brightness profile fitting methods, and the host-galaxy Petrosian magnitudes were measured using an automated pipeline identical to the SDSS one. The stellar mass for each galaxy was measured using the KCORRECT package. Of the 185 galaxies in K11, 38 of them are "lowmass" galaxies with $M_{\star}<10^{10} M_{\odot}$. For this work, we discard the six galaxies with more than $50 \%$ AGN contribution at $r$ band to avoid the selection of galaxies with uncertain stellar masses. The median $r$-band absolute magnitude for the rest of the 32 low-mass AGNs is -20.11, which is only slightly lower than the median of our NuSTAR sample $(-20.03)$. To test whether the $M_{\star}$ estimated in K11 is systematically different from the $M_{\star}$ of our NUSTAR sample estimated using the SEDfitting approach, we obtain the optical to mid-IR photometry 
for K11 objects within the SDSS footprint and use the SEDfitting method described in Section 3.1 to recalculate their $M_{\star}$. We find that the $M_{\star}$ measured using our SED-fitting approach is slightly lower than the $\mathrm{K} 11 M_{\star}$ by a median value of 0.09 dex. With the much larger $\approx 0.3$ dex uncertainty caused by the stellar population synthesis model degeneracy (e.g., Conroy et al. 2009), we consider the $M_{\star}$ of the K11 low-mass AGNs and our NUSTAR sample to be directly comparable.

For the hard X-ray luminosity, we match the K11 AGNs with the Swift/BAT 70-month catalog and convert the $14-195 \mathrm{keV}$ luminosity provided in the 70-month catalog to $10-40 \mathrm{keV}$ luminosity assuming a typical AGN spectrum with a photon index of $\Gamma=1.8$. The correction is $\approx 0.4 \mathrm{dex}$ for the redshift range of the K11 sample. The column densities for the K11 AGNs are culled from C. Ricci et al. (2017, in preparation), which analyzes the soft X-ray spectra of all BAT AGNs using archival data (also see Ricci et al. 2015). The key properties of the K11 low-mass AGNs are summarized in Table 6.

\section{References}

Alam, S., Albareti, F. D., Prieto, C. A., et al. 2015, ApJS, 219, 12 Alexander, D. M., Chary, R. R., Pope, A., et al. 2008, ApJ, 687, 835 Alexander, D. M., Stern, D., Del Moro, A., et al. 2013, ApJ, 773, 125 Asmus, D., Gandhi, P., Hönig, S. F., Smette, A., \& Duschl, W. J. 2015 , MNRAS, 454, 766

Assef, R. J., Kochanek, C. S., Brodwin, M., et al. 2008, ApJ, 676, 286 Assef, R. J., Kochanek, C. S., Brodwin, M., et al. 2010, ApJ, 713, 970 Assef, R. J., Stern, D., Kochanek, C. S., et al. 2013, ApJ, 772, 26 Baldassare, V. F., Reines, A. E., Gallo, E., et al. 2016, ApJ, 829, 57 Baldassare, V. F., Reines, A. E., Gallo, E., \& Greene, J. E. 2015, ApJL, 809, L14

Baldassare, V. F., Reines, A. E., Gallo, E., \& Greene, J. E. 2017, ApJ, 836, 20 Baldwin, J. A., Phillips, M. M., \& Terlevich, R. 1981, PASP, 93, 5 Baloković, M., Matt, G., Harrison, F. A., et al. 2015, ApJ, 800, 62 Barth, A. J., Greene, J. E., \& Ho, L. C. 2005, ApJL, 619, L151 Barth, A. J., Greene, J. E., \& Ho, L. C. 2008, AJ, 136, 1179 Baumgartner, W. H., Tueller, J., Markwardt, C. B., et al. 2013, ApJS, 207, 19 Begelman, M. C., \& Rees, M. J. 1984, MNRAS, 206, 209

Bentz, M. C., Denney, K. D., Grier, C. J., et al. 2013, ApJ, 767, 149

Bertin, E., \& Arnouts, S. 1996, A\&AS, 117, 393

Bianchi, L., Conti, A., \& Shiao, B. 2014, AdSpR, 53, 900

Bianchi, S., Corral, A., Panessa, F., et al. 2008, MNRAS, 385, 195

Blanton, M. R., Brinkmann, J., Csabai, I., et al. 2003, AJ, 125, 2348

Bonnarel, F., Fernique, P., Bienaymé, O., et al. 2000, A\&AS, 143, 33 Cameron, E. 2013, PASA, 28, 128

Cardelli, J. A., Clayton, G. C., \& Mathis, J. S. 1989, ApJ, 345, 245 Cash, W. 1979, ApJ, 228, 939

Chary, R., \& Elbaz, D. 2001, ApJ, 556, 562

Chen, C.-T. J., Hickox, R. C., Alberts, S., et al. 2015, ApJ, 802, 50

Chen, C.-T. J., Hickox, R. C., Goulding, A. D., et al. 2017, ApJ, in press (arXiv:1701.05207)

Chung, S. M., Kochanek, C. S., Assef, R., et al. 2014, ApJ, 790, 54

Civano, F., Hickox, R. C., Puccetti, S., et al. 2015, ApJ, 808, 185

Civano, F., Mignoli, M., Comastri, A., et al. 2007, A\&A, 1233, 1223

Comastri, A., Mignoli, M., Ciliegi, P., et al. 2002, ApJ, 571, 771

Conroy, C., Gunn, J. E., \& White, M. 2009, ApJ, 699, 486

Darling, J. 2014, Chandra Propos. ID \#16620657

Del Moro, A., Alexander, D. M., Bauer, F. E., et al. 2015, MNRAS, 456, 2105

Dong, R., Greene, J. E., \& Ho, L. C. 2012a, ApJ, 761, 73

Dong, X.-B., Ho, L. C., Yuan, W., et al. 2012b, ApJ, 755, 167

Donley, J. L., Koekemoer, A. M., Brusa, M., et al. 2012, ApJ, 748, 142

Eckart, M. E., McGreer, I. D., Stern, D., Harrison, F. A., \& Helfand, D. J. 2010, ApJ, 708, 584

Elitzur, M., Ho, L. C., \& Trump, J. R. 2014, MNRAS, 438, 3340

Elitzur, M., \& Netzer, H. 2016, MNRAS, 459, 585

Elvis, M., Schreier, E., Tonry, J., Davis, M., \& Huchra, J. 1981, ApJ, 246, 20

Fazio, G. G., Hora, J. L., Allen, L. E., et al. 2004, ApJS, 154, 10

Feigelson, E. D., \& Nelson, P. I. 1985, ApJ, 293, 192

Fiore, F., Puccetti, S., Brusa, M., et al. 2009, ApJ, 693, 447
Gallo, E., Treu, T., Jacob, J., et al. 2008, ApJ, 680, 154

Gandhi, P., Horst, H., Smette, A., et al. 2009, A\&A, 502, 457

Gandhi, P., Lansbury, G. B., Alexander, D. M., et al. 2014, ApJ, 792, 117

Gehrels, N. 1986, ApJ, 303, 336

Gordon, K. D., \& Clayton, G. C. 1998, ApJ, 500, 816

Greene, J., \& Ho, L. 2005, ApJ, 630, 122

Greene, J. E. 2012, NatCo, 3, 1304

Greene, J. E., \& Ho, L. C. 2004, ApJ, 610, 722

Greene, J. E., \& Ho, L. C. 2007a, ApJ, 656, 84

Greene, J. E., \& Ho, L. C. 2007b, ApJ, 670, 92

Greenhill, L. J., Tilak, A., \& Madejski, G. 2008, ApJL, 686, L13

Grier, C. J., Martini, P., Watson, L. C., et al. 2013, ApJ, 773, 90

Griffith, R. L., Tsai, C.-W., Stern, D., et al. 2011, ApJL, 736, 18

Hainline, K. N., Reines, A. E., Greene, J. E., \& Stern, D. 2016, ApJ, 832, 119

Hambly, N., MacGillivray, H., Read, M., et al. 2001, MNRAS, 326, 1279

Harrison, F. A., Craig, W. W., Christensen, F. E., et al. 2013, ApJ, 770, 103

Hopkins, P. F., Hickox, R., Quataert, E., \& Hernquist, L. 2009, MNRAS, 398, 333

Izotov, Y. I., Guseva, N. G., Fricke, K. J., Krügel, E., \& Henkel, C. 2014, A\&A, 570, A97

Kauffmann, G., Heckman, T. M., Tremonti, C., et al. 2003, MNRAS, 346,1055

Kelvin, L. S., Driver, S. P., Robotham, A. S. G., et al. 2014, MNRAS, 439,1245

Kewley, L. J., Groves, B., Kauffmann, G., \& Heckman, T. 2006, MNRAS, 372,961

Kormendy, J., \& Ho, L. C. 2013, ARA\&A, 51, 511

Koss, M., Mushotzky, R., Veilleux, S., et al. 2011, ApJ, 739, 30

Lacy, M., Storrie-Lombardi, L. J., Sajina, A., et al. 2004, ApJS, 154, 166

LaMassa, S. M., Ricarte, A., Glikman, E., et al. 2016, ApJ, 820, 70

Lansbury, G. B., Alexander, D. M., Moro, A. D., et al. 2014, ApJ, 785, 17

Lansbury, G. B., Gandhi, P., Alexander, D. M., et al. 2015, ApJ, 809, 115

Lansbury, G. B., Stern, D., Aird, J. A., et al. 2017, ApJ, 836, 99

Lehmer, B. D., Tyler, J. B., Hornschemeier, A. E., et al. 2015, ApJ, 806, 126

Lemons, S. M., Reines, A. E., Plotkin, R. M., Gallo, E., \& Greene, J. E. 2015, ApJ, 805, 12

Ludlam, R. M., Cackett, E. M., Gultekin, K., et al. 2015, MNRAS, 447, 2112

Lusso, E., Hennawi, J. F., Comastri, A., et al. 2013, ApJ, 777, 33

Makovoz, D., \& Marleau, F. R. 2005, PASP, 117, 1113

Martin, D. C., Fanson, J., Schiminovich, D., et al. 2005, ApJL, 619, L1

Masini, A., Comastri, A., Baloković, M., et al. 2016, A\&A, 589, A59

Mateos, S., Alonso-Herrero, A., Carrera, F. J., et al. 2013, MNRAS, 434, 941

Mateos, S., Carrera, F. J., Alonso-Herrero, A., et al. 2015, MNRAS, 449, 1422

Merloni, A., Bongiorno, A., Brusa, M., et al. 2014, MNRAS, 437, 3550

Merloni, A., Predehl, P., Becker, W., et al. 2012, arXiv:1209.3114

Mezcua, M., Civano, F., Fabbiano, G., Miyaji, T., \& Marchesi, S. 2016, ApJ, 817,20

Miller, B. P., Gallo, E., Greene, J. E., et al. 2015, ApJ, 799, 98

Moran, E. C., Filippenko, A. V., \& Chornock, R. 2002, ApJL, 579, L71

Moran, E. C., Shahinyan, K., Sugarman, H. R., Vélez, D. O., \& Eracleous, M. 2014, AJ, 148, 136

Mukherjee, E. S., Walton, D. J., Bachetti, M., et al. 2015, ApJ, 808, 64

Mullaney, J. R., Del-Moro, A., Aird, J., et al. 2015, ApJ, 808, 184

Narayan, R., Yi, I., \& Mahadevan, R. 1995, Natur, 374, 623

Onken, C., Ferrarese, L., Merritt, D., et al. 2004, ApJ, 615, 645

Pardo, K., Goulding, A. D., Greene, J. E., et al. 2016, ApJ, 831, 203

Park, T., Kashyap, V. L., Siemiginowska, A., et al. 2006, ApJ, 652, 610

Plotkin, R. M., Gallo, E., Haardt, F., et al. 2016, ApJ, 825, 139

Reines, A., \& Comastri, A. 2016, PASA, in press (arXiv:1609.03562)

Reines, A., Plotkin, R., Russell, T., et al. 2014, ApJL, 787, L30

Reines, A. E., Greene, J. E., \& Geha, M. 2013, ApJ, 775, 116

Reines, A. E., Reynolds, M. T., Miller, J. M., et al. 2016, ApJL, 830, L35

Reines, A. E., Sivakoff, G. R., Johnson, K. E., \& Brogan, C. L. 2011, Natur, 470, 66

Reines, A. E., \& Volonteri, M. 2015, ApJ, 813, 82

Ricci, C., Bauer, F. E., Arevalo, P., et al. 2016, ApJ, 820, 5

Ricci, C., Ueda, Y., Koss, M. J., et al. 2015, ApJL, 815, L13

Rigby, J., Rieke, G., Pérez-González, P., et al. 2005, ApJ, 627, 134

Sartori, L. F., Schawinski, K., Treister, E., et al. 2015, MNRAS, 454, 3722

Satyapal, S., Secrest, N. J., McAlpine, W., et al. 2014, ApJ, 784, 113

Satyapal, S., Secrest, N. J., Rothberg, B., et al. 2016, ApJ, 827, 58

Schawinski, K., Koss, M., Berney, S., \& Sartori, L. 2015, MNRAS, 451, 2517

Schlegel, D. J. D., Finkbeiner, D. P. D., \& Davis, M. 1998, ApJ, 500, 525

Schramm, M., Silverman, J. D., Greene, J. E., et al. 2013, ApJ, 773, 150

Secrest, N. J., Satyapal, S., Gliozzi, M., et al. 2015, ApJ, 798, 38

Simmonds, C., Bauer, F. E., Thuan, T. X., et al. 2016, A\&A, 596, A64 
Skrutskie, M. F., Cutri, R. M., Stiening, R., et al. 2006, AJ, 131, 1163 Stern, D. 2015, ApJ, 807, 129

Stern, D., Assef, R. J., Benford, D. J., et al. 2012, ApJ, 753, 30

Stern, D., Eisenhardt, P., Gorjian, V., et al. 2005, ApJ, 631, 163

Stern, D., Lansbury, G. B., Assef, R. J., et al. 2014, ApJ, 794, 102

Takada, M., Ellis, R., Chiba, M., et al. 2012, PASJ, 66, R1

Trump, J. R., Nagao, T., Ikeda, H., et al. 2011, ApJ, 732, 23

Trump, J. R., Sun, M., Zeimann, G. R., et al. 2015, ApJ, 811, 26

Vasudevan, R. V., \& Fabian, A. C. 2007, MNRAS, 381, 1235

Volonteri, M. 2010, A\&ARv, 18, 279
Walton, D. J., Harrison, F. A., Grefenstette, B. W., et al. 2014, ApJ, 793, 21 Wright, E. L., Eisenhardt, P. R. M., Mainzer, A. K., et al. 2010, AJ, 140, 1868 Wyder, T. K., Martin, D. C., Schiminovich, D., et al. 2007, ApJS, 173, 293 Xiao, T., Barth, A. J., Greene, J. E., et al. 2011, ApJ, 739, 28

Xue, Y. Q., Wang, S. X., Brandt, W. N., et al. 2012, ApJ, 758, 129

York, D., Adelman, J., Anderson, J., Jr., et al. 2000, AJ, 120, 1579

York, D. G., Khare, P., Vanden Berk, D., et al. 2006, MNRAS, 367, 945

Yuan, F., \& Narayan, R. 2004, ApJ, 612, 724

Zhang, J. S., Henkel, C., Kadler, M., et al. 2006, A\&A, 944, 16

Zibetti, S., Charlot, S., \& Rix, H.-W. 2009, MNRAS, 400, 1181 